\title{
Exploring perspectives on masculinity and mental wellbeing: The case of students in higher education in New Zealand
}

\author{
By
}

Ellis Louise Read

\author{
A thesis \\ submitted to Victoria University of Wellington \\ in partial fulfilment of the requirements for the degree of \\ Master of Commerce
}

Victoria University of Wellington 


\begin{abstract}
This study sits at the intersection of research on mental wellbeing, masculinity and higher education. Men's mental wellbeing has been a focal point for academic research in recent years, as has the mental wellbeing of higher education students. However, there is a paucity of research that explores this topic in the New Zealand context. This study explores perceptions of masculinity and mental wellbeing by male students in New Zealand tertiary institutions. It considers the ways in which masculinity may both constrain and enable mental wellbeing. The research adopts a cultural studies approach which focuses on values and practices that participants perceive to be associated with masculinity. Twelve in-depth interviews were used to gather rich insights into this topic. The findings suggest a persistence of a cultural script across young men in New Zealand. The most prominent themes identified surrounded sport and drinking, findings which support earlier works on masculinity in New Zealand. It was also found that, on the one hand, the devaluation of emotion, high levels of competition and intergroup hierarchy constrain mental wellbeing. Whilst on the other hand, mental wellbeing is facilitated through the social bonding that shared masculine practices and values provide. In closing, the thesis outlines implications for future research and practice.
\end{abstract}




\section{Acknowledgements}

First and foremost, I would like to thank my research supervisor Dr Sally Riad, who provided me with ongoing support and guidance throughout this past year. I will be forever grateful for the knowledge, inspiration and kind words that you have shared with me; without which this thesis would likely not have been possible.

Thank you to my friends and family. All of you have provided me with different types of support and in your own ways you have each been integral to the completion of this work. Saloni, Adam and Sam, thank you for "riding the wave" with me, I am glad that I got to share this experience with such amazing people. Sky and Ria, thank you for your support, words of encouragement and academic advice. Dad, Angela, Mum and Mark, thank you for always believing in me - no matter what path I choose to take.

Lastly, thank you to all the students who participated in this study. I feel privileged to share your valuable and eye-opening contributions to this topic. 


\section{Contents}

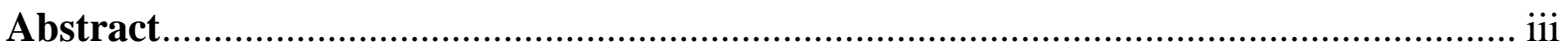

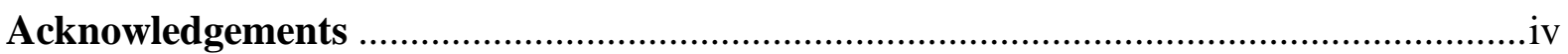

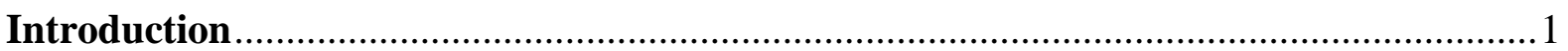

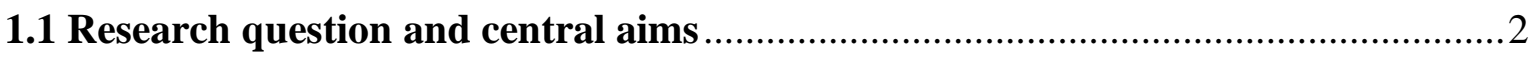

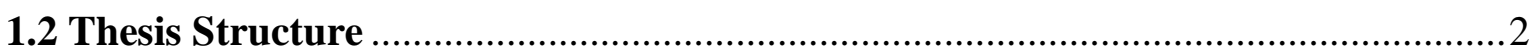

Literature review

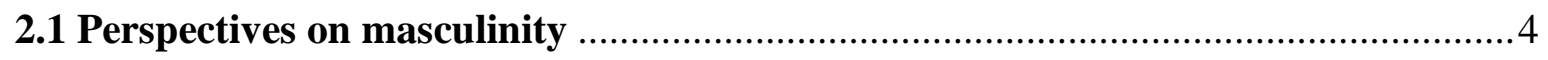

2.1.1 Biological essentialism ............................................................................ 4

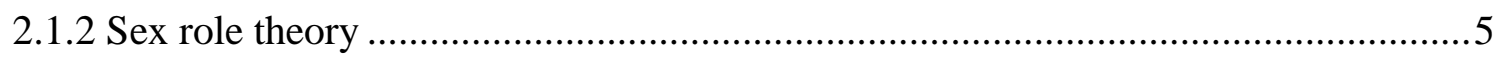

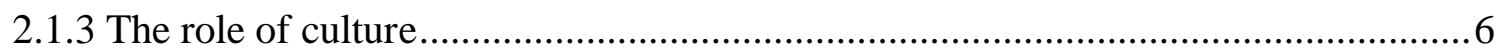

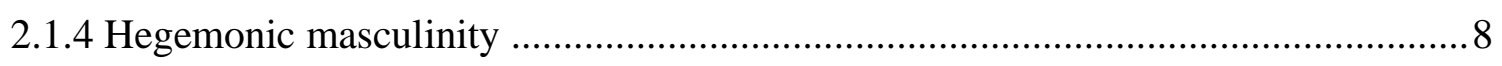

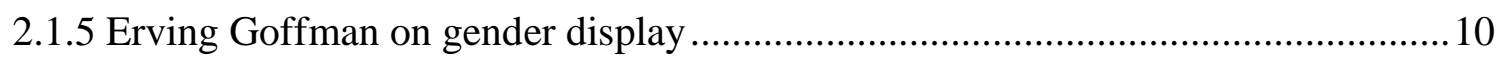

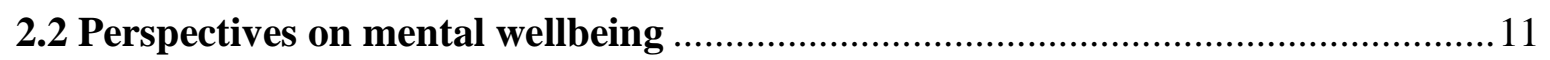

2.2.1 Concern for the mental wellbeing of men........................................................ 12

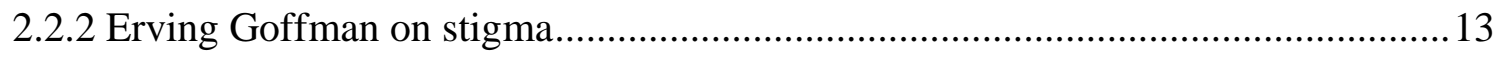

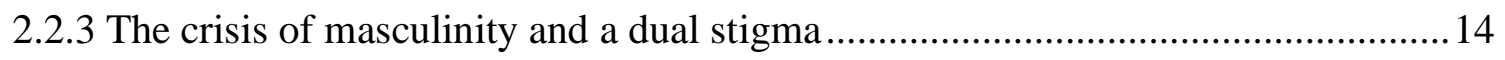

2.3.4 A focus on positive forms of masculinity ........................................................ 15

2.3.5 Mental wellbeing of male students in higher education ..................................... 16

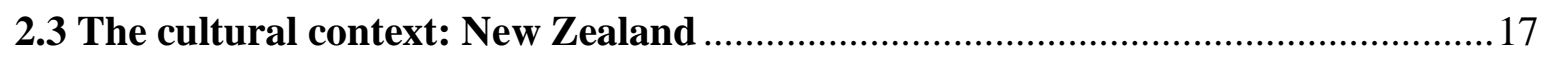

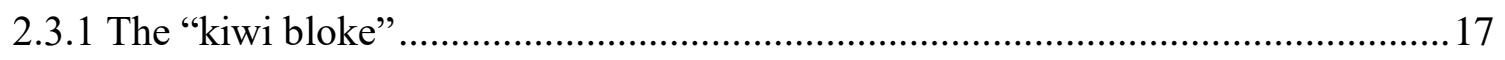

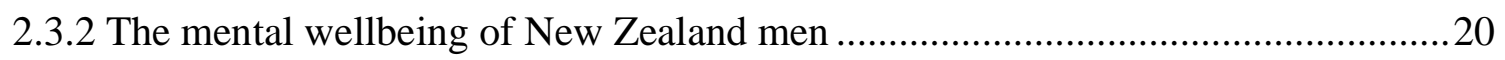

2.3.3 Mental wellbeing and male university students in New Zealand ..........................21

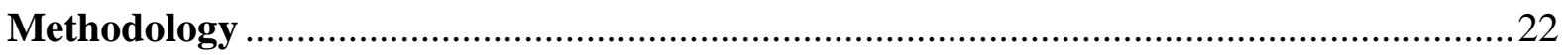

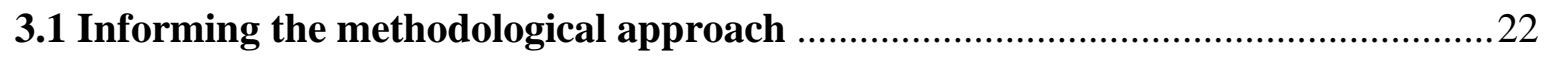

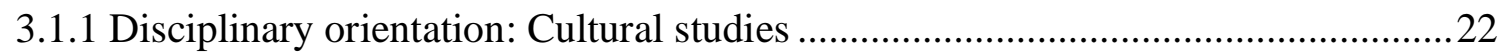

3.1.2 Theoretical perspectives of Erving Goffman and R.W Connell ............................24

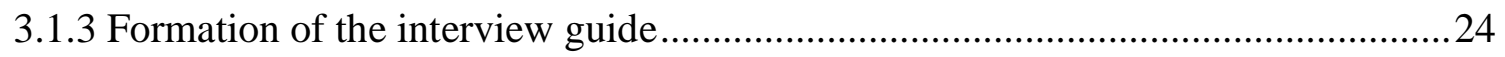

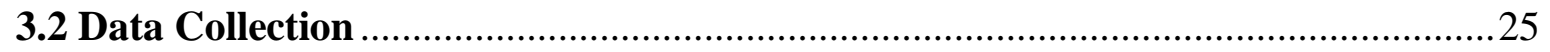

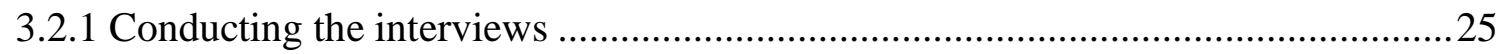

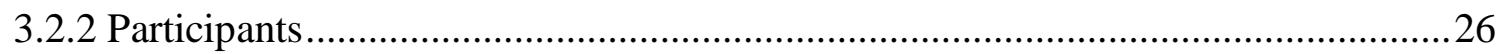

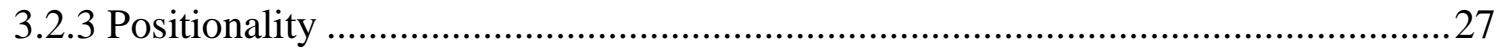

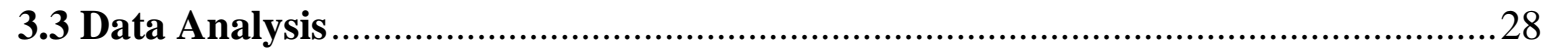

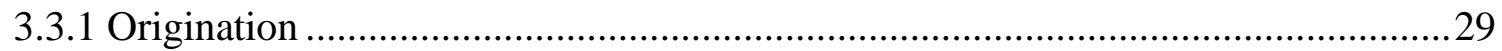

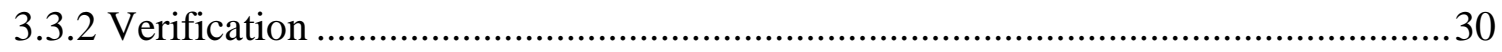


3.3.3 Nomination .31

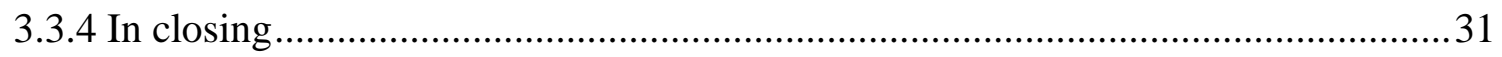

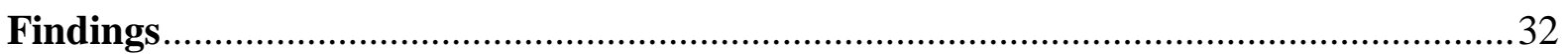

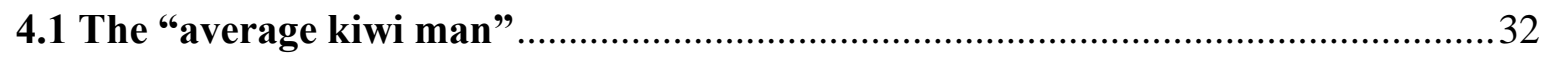

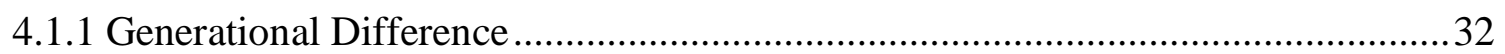

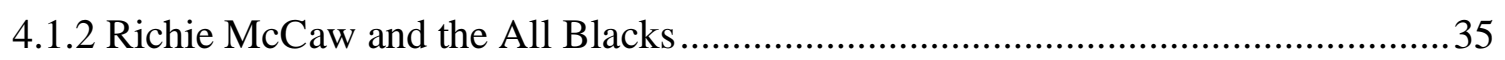

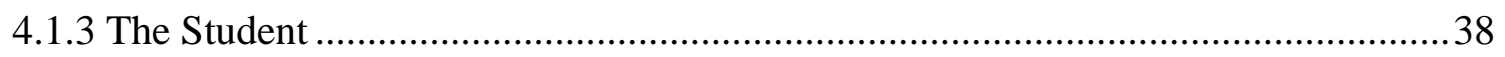

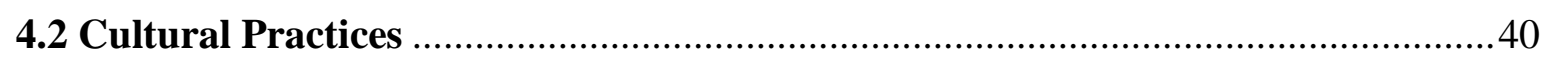

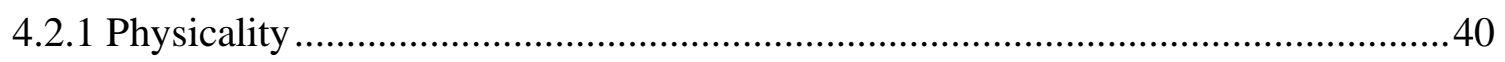

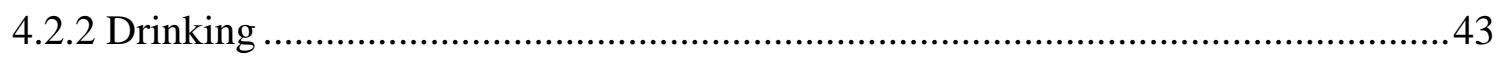

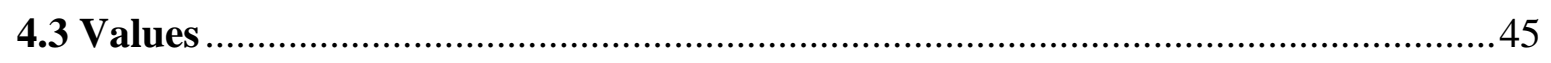

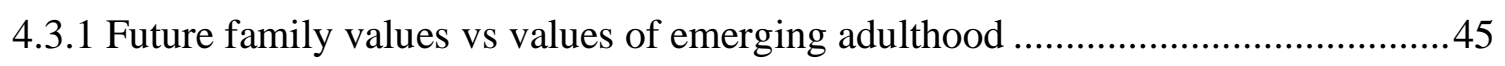

4.3.2 Loyalty between men vs Self reliance ..............................................................4

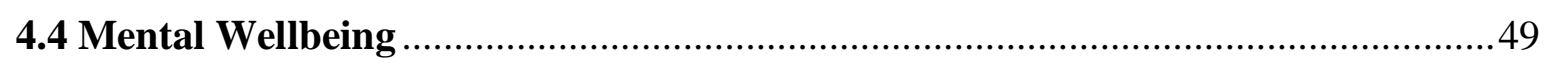

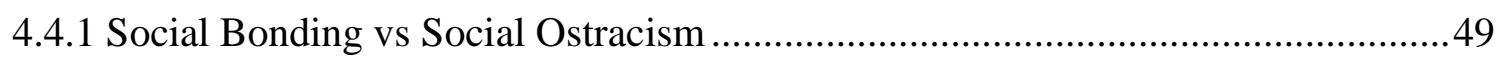

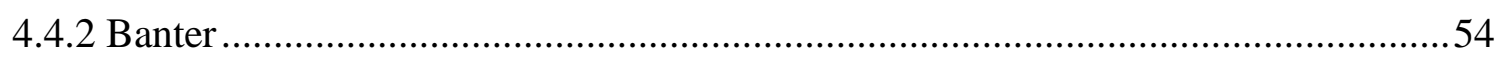

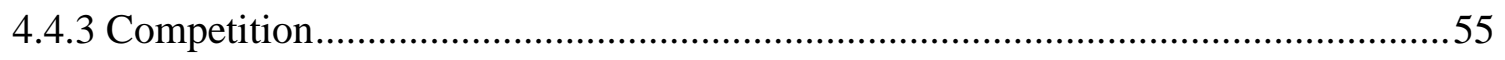

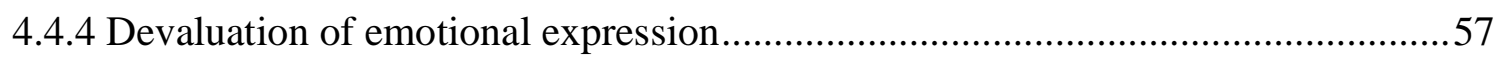

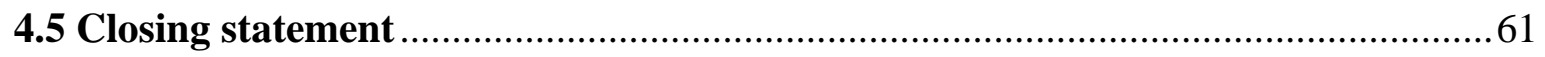

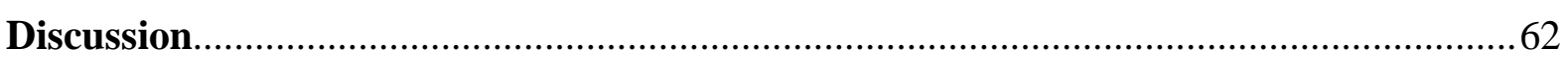

5.2 The persistence of a cultural script for New Zealand men ...................................62

5.2.1 The "kiwi bloke": a cultural echo or a relived experience? ...................................63

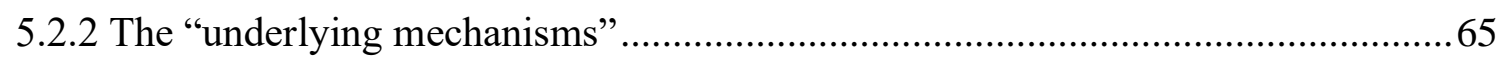

5.2.3 The impacts of the cultural script on male students in higher education ................67

5.2.4 The convergence of the life-course and the "masculine script" ............................68

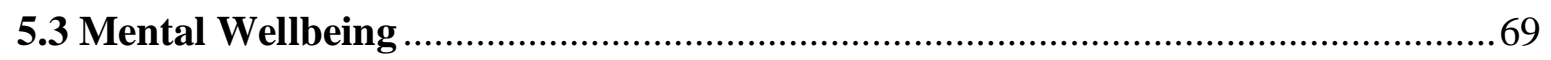

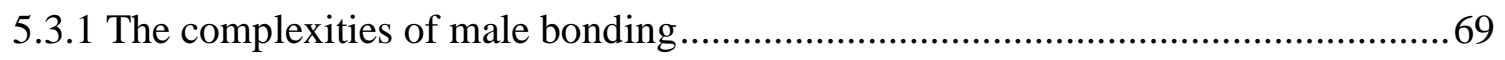

5.3.2 Male bonding in the context of higher education ............................................... 71

5.3.2 The ambiguous line between healthy and toxic ................................................ 71

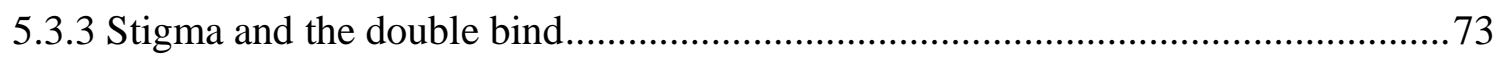

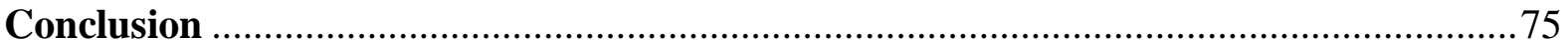

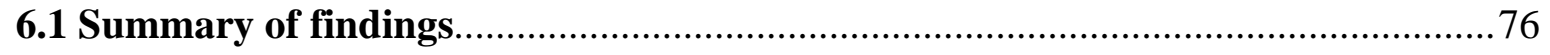

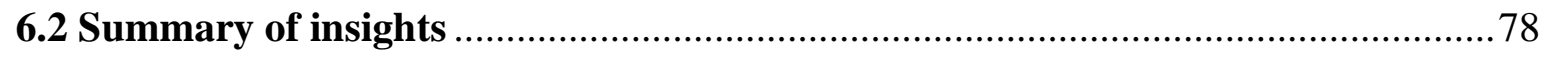

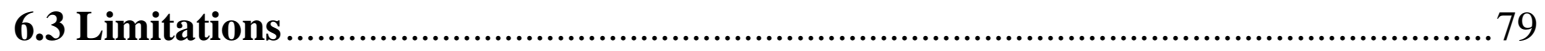

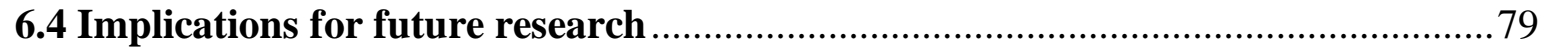




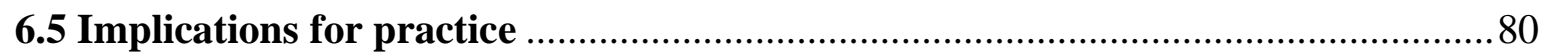

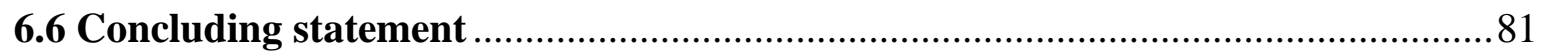

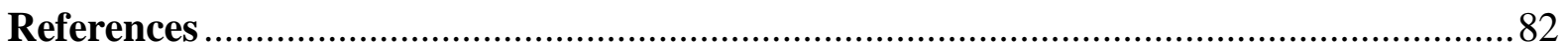

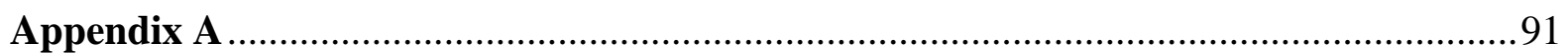

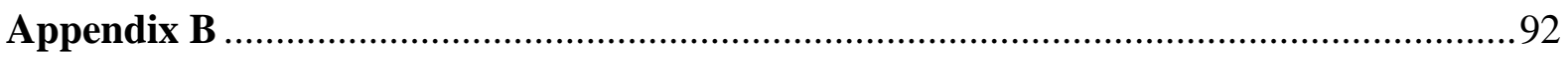

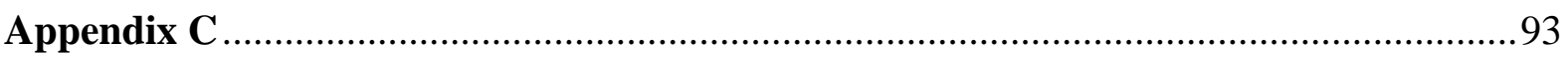

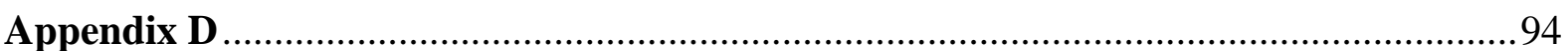




\section{Chapter 1}

\section{Introduction}

The old idea of manhood has grown obsolete, and the new is still invisible to us, and we grope after it in darkness, one clutching this phantom (Thomas Carlyle, 1831, as cited in Adams, 2018, p.1)

Thomas Carlyle highlights changing perceptions of masculinity that were taking place during his time in the nineteenth century. Almost two hundred years later, the discussion surrounding what it means to be a man continues (Lomas, 2013; Wassersug \& Hamilton, 2018). Previous literature has highlighted a specific concern for the ways in which masculinity affects the mental wellbeing of men (Pitman et al., 2012; Mckenzie, 2017; Oliffe \& Phillips, 2008). The concept of mental wellbeing extends beyond the simple absence of mental illness. It encompasses a range of aspects that determine our ability to enjoy life and contribute productively to our societies (Barden \& Caleb, 2019). This study explores perceptions of masculinity and the ways in which it may enable or constrain mental wellbeing as pertains to university students.

The research adopts a cultural studies approach, which focuses on the values and practices that are associated with masculinity. The theoretical work by R.W Connell (Connell, 1985, 1997, 2005; Connell \& Messerschmidt, 2005) and Erving Goffman (1976, 1959) inform a perspective on masculinity as enactment. From this perspective, masculinity is a fluid social construct influenced by specific cultures and histories. The cultural context of this study is New Zealand, a country with unique masculine ideals. Over the past year, the mental wellbeing of men has been repeatedly discussed within the New Zealand media (Kightley, 2018; Skerrett, 2019; Marvelly, 2019). There are several initiatives which seek to address the concern for the wellbeing of New Zealand men, such as "Farmstrong" (Skerrett, 2019) and the national rugby teams" "vulnerability group" (Marvelly, 2019). These programs are based in specific organizational contexts and aim to encourage men to engage with their mental wellbeing.

However, there is a paucity of both academic research and practical initiatives that address the mental wellbeing of male students in New Zealand higher education institutions. Awareness of the importance of the mental wellbeing of tertiary students is rising (Baik et 
al., 2019; Storrie et al., 2010). Last year, university students in New Zealand voiced the need for more mental health support (Long, 2018). The transition from high school to further education presents many challenges and adjustments. Overwhelming feelings and fear of failure are not uncommon experiences for university students ("Tertiary student leaders", 2018), which means a strong sense of resilience is needed to ensure mental wellbeing flourishes in this environment. It has been recognized that universities are well-placed to address mental wellbeing concerns (Baik et al., 2019), however, information is needed to inform effective practices and programs. This study seeks to address this gap in the literature by providing insight into male students' perceptions of masculinity and mental wellbeing in New Zealand.

\subsection{Research question and central aims}

The research question that guides this study is: How does masculinity shape the ways in which male students perceive mental wellbeing? The following sub questions were developed to address the perspectives that this research will explore:

1) How does masculinity enable constructive views on mental wellbeing?

2) How does masculinity constrain views on mental wellbeing?

The primary aim is to contribute to knowledge at the intersection of masculinity, mental wellbeing and higher education. As a secondary aim, the findings would also inform practices conducive to student wellbeing in tertiary organizations.

\subsection{Thesis Structure}

This thesis is organised into six chapters including the introduction.

Chapter 2 - Literature Review:

This chapter reviews the relevant literature on masculinity and mental wellbeing. It begins with an overview of perspectives on masculinity and details the theoretical perspectives that ground this research. The chapter then offers an overview of mental wellbeing with a subsequent focus on men's mental wellbeing research. This chapter concludes with a discussion of the relevance of these perspectives to the cultural context of New Zealand

Chapter 3 - Methodology

This chapter describes the methodological approach used for this study. The research design is qualitative and exploratory premised in cultural studies. The core focus is perceived 
cultural values and practices. The study included twelve in-depth interviews to gain insight into the research question and respective sub-questions. The chapter concludes by detailing the steps taken to analyse and categorize the data.

Chapter 4 - Findings

This chapter details the findings which stemmed from the data analysis process. It is organised across four structural themes that are as follows: the "average kiwi man", cultural practices, values and mental wellbeing. The respective sub-themes provide insights into each theme.

Chapter 5 - Discussion

The discussion chapter provides a synthesis of the research findings and the literature that informed this study. Due to the inductive design of this study, the chapter also theorizes the findings to provide further insight.

Chapter 6 - Conclusion

This chapter offers a closing statement followed by summaries of the findings and insights. It concludes with implications for further research and practice. 


\section{Chapter 2}

\section{Literature review}

Reviewing the research on masculinity and mental wellbeing can be a challenging task due to the range of perspectives that are available on both topics within the academic literature. Therefore, it is important to establish the position that will be taken within the current research. This chapter is divided into three sections. The first section provides a brief overview of the various perspectives across research on masculinity. Subsequently the perspective that underpins this research is detailed, along with the insights of R.W Connell and Erving Goffman, the two primary theoretical perspectives in this study. The second section begins with an overview of the literature on mental wellbeing. It also outlines the relevance of mental wellbeing to both masculinity and higher education institutions. The last section discusses the relevance of the above topics to the cultural context of New Zealand.

\subsection{Perspectives on masculinity}

There is an important distinction between the term's "sex" and "gender". Although in some contexts the terms are still used interchangeably, the distinction between them was made in 1955 by John Money (Udry, 1994; Kirkpatrick, 2003). Prior to this "gender" was a word primarily used within linguistics to indicate the feminine or masculine nouns and adjectives in non-English languages (Kirkpatrick, 2003). By Money's distinction, the term "sex" should be used to refer to someone's biological classification of male or female, which is assigned at birth via physical characteristics or via chromosome analysis. The term "gender" should be used in relation to differences in behaviour by sex. Therefore, gender is a term which encompasses a more complex dynamic of variables, such as cultural, social, biological and psychological. The question of how gender is comprised and influences our behaviour is the subject of interdisciplinary debate. As Wassersug \& Hamilton (2018, p.153) state; "an ongoing, fundamental question is: what is masculinity, and how is it expressed?"

\subsubsection{Biological essentialism}

Physical differences between men and women are largely apparent, however to what extent our physical sex interacts with our gender is still debated. Scientific inquisition into the extent of physiological interaction between our physical sex and sex-differentiated behaviour is ongoing. There are decades worth of findings which stem from differences in brain physiology and neuroimaging that suggest innate differences, for example; in areas such as 
competitiveness, aggression and risk behaviour (Stern, 2016). From this biological perspective, without the influence of sociological factors, if everyone was given the same basis to reflect individuality there would still be significant differences in behaviour between the sexes due to variations in anatomy and physical abilities (Stern, 2016). The work in this area is largely based on chromosomal, hormonal and genetic information. It is also informed by an evolutionary perspective, which posits that sex differences in psychological and behavioural tendencies can be likened to that of Darwin's natural selection (Eckes \& Trautner, 2012).

The biological perspective and social perspectives which inform research on masculinity are often dichotomized (Wassersug \& Hamilton, 2018). The ingrained focus on figuring out the innate qualities of individuals at the statistically significant level is a key imperative for those pursuing scientific enquiry. Some view this as biological essentialism, which problematically simplifies "typical" male behaviour to just "the way men are" (Lomas, 2013). It is perhaps more so problematic because of the weight and influence scientific enquiry has had on popular culture when it comes to sex differences in behaviour i.e. Men are from Mars, Women are from Venus (Lomas, 2013). This is problematic because it has reinforced a widely held assumption that masculinity is something which is fixed to biology and therefore creates a container to which all "normal" men should fit within (Beynon, 2001). This does not mean that our physiology is not linked to the social construction of masculinity, rather we do not know the nature of the interaction in its entirety (Beynon, 2001). Generally, it is now excepted within academic literature that our gendered behaviour is a mixture of physiological, cultural, economic and social factors.

\subsubsection{Sex role theory}

One of the prevailing sociological perspectives on masculinity and gender throughout the 20th century was sex-role theory (McKenzie, 2017; Courtenay, 2000). Although it is a theory on socialisation it has been a dominant theory for informing psychological literature on sex differences and is entwined with biological sex (Pleck, 2018). The central premise is also deemed "essentialist" in nature and although it sets itself apart from innate biological perspectives on gender it proposes that masculinity or femininity is something that is internalised within the individual (Smiler, 2004). Men and women attempt to acquire attributes, traits or interests that psychologically affirm their biological sex (Pleck, 2018). If these are not affirmed, then the theory proposes that individuals may suffer from ill adjustment and diminished well-being (Pleck, 2018). 
This perspective has since been heavily criticized. Most notedly by Raewyn Connell who viewed this theoretical ideology to be leaving questions of power and change unanswered (Demetriou, 2013). Role theory in general has been criticized as being too normative and assuming a concrete reality. By aligning itself with biological sex, sex role theory puts emphasis on the binary of gender and then establishes a neutral set of behaviours that align with the sexes. This means that things such as race, ethnicity, sexuality and even age are invisible considerations. Deviation from normative behaviour is considered within sex role theory, however they are deemed role conflicts and not just a different variation of living. What is not considered in-depth is how the role expectations become applied (Connell, 1985). It is assumed that expectations and norms associated with the sex role are celebrated when an individual conforms to them and those individuals would be punished if they deviate. But why are these traits celebrated or punished? Connell (1985) argues that, without explanation for this question, the theory dissolves into voluntarism and into the assumption that people are choosing to maintain these customs.

The roles, expectations and norms attached to biological sex through sex role theory do not explain the extensive and complex ways gender can be inconsistently enacted upon through culture. For example, characteristics and expectations that are "robust" sex differences like "aggressiveness" and "softness" are relative and not mutually exclusive. An example of a shift in the display of gendered traits can be observed in the reformation of masculinity in eighteenth century England (Capdeville, 2012). The establishment of clubs during this period sought to redefine masculinity by taking influence from "effeminate" French culture and replacing "roughness" with refined politeness and polished dress (Capdeville, 2012). This gave rise to the term "gentleman" and is an illustration of the influence of society and culture in shaping how masculinity can be displayed. Connell (1997) has noted that there are times even in contemporary western history where the concept that men supress displays of emotion did not apply at all. Which is a particularly important notion regarding this current research.

\subsubsection{The role of culture}

The present study adopts an interdisciplinary cultural studies approach. The use of the term "culture" is not just aligned with our notions of national culture, although this will be discussed in relation to masculinity in New Zealand. Instead, the term is aligned with an anthropological view of culture in that it is "any expressive activity which contributes to social learning" (Agger, 2014 p.2). This perspective has also been taken by Beynon in his 
book Masculinities and Culture where he states that he must "side-line perspectives associated with socio-biology, evolutionary psychology and which tend to 'naturalize' male behaviour" (2001, p.3).There is a focus on how contextual and public social arrangements are lived and made sense of at the subjective level (Pickering, 2008).

One of the founders of cultural studies, Raymond Williams, has argued that there is no such thing as the masses, only ways of seeing people as masses (Sardar, 2004). It is the analysis of the popular and how this interacts with the practices, values, languages and logic of people in their every-day lives that should be of focus (Nelson et al., 1992). The emphasis of this approach is to focus on what people $d o$, which may vary across cultures and generations. In order to employ a cultural studies approach there needs to be a clear focus on the elements of culture that will be explored. Therefore, this study maintains a focus on values and practices that are associated with masculinity and mental wellbeing. Further detail will be given within the methodology section of this thesis regarding the integration of values and practices into the interview process. However, an outline of these core terms will be detailed below.

Value theory, in its broadest sense, applies to large areas of philosophy which is concerned with how things should or should not be. Exploring values means investigating which things are good or bad, what makes them good or bad and how good or bad something is (Hirose \& Olson, 2015). Therefore, there is an evaluative aspect, as values can serve as standards or criteria (Schwartz, 2012). They are relative to one another and form an order of importance. Values can perhaps be seen to precede our practices, as they can guide behaviour across multiple settings or situations (Schwartz, 2012). For instance; honesty is a value that can span across school, work, home and social life. However, values are something which may be difficult to explicitly identify when compared to practices.

Our actions and practices can be an expression of our values in multiple ways. Practices are what we $d o$ and can be anything from collective practices (i.e. playing group sport, singing hymns at church) to how we conduct ourselves individually (i.e. bowing, shouting, crying). What we do is often influenced by, or in conflict with, values that are held within our broader social worlds. Whilst individual experience is of importance when taking a cultural studies approach, that experience cannot be understood without reference to conceptual and theoretical ideas (Pickering, 2008). To conceptualise the values and practices associated with 
masculinity in this study I will be drawing on the works of R.W Connell (Connell, 1985, 1997, 2005; Connell \& Messerschmidt, 2005) and Erving Goffman (1976,1959).

\subsubsection{Hegemonic masculinity}

One of the most significant contributions to the academic field of masculinity is the concept of hegemonic masculinity which stems from the work of R.W Connell (Beynon, 2001). This concept has been used extensively within studies relevant to masculinity in New Zealand (Bannister, 2005; Sim \& Thomson, 2000; Gee \&Jackson, 2012). It has also been used to study masculinity in educational settings and men's difficulty in responding to disabilities or injury (Connell \& Messerschmidt, 2005). In its simplest explanation, hegemonic masculinity is the most idealised form of masculinity at any given place or time (Courtenay, 2000). This idealised form can be brutally enforced, however it can also be negotiated upon and reinforced through power and achievement (Beynon, 2001). It looks at patterns of practice, therefore things done, instead of looking at a set of role expectations or internal identity (Connell \& Messerschmidt, 2005).

There are some important distinctions to be made concerning the use of the concept within the context of this study. Hegemonic masculinity was developed in critique of sex role theory, which as previously discussed, reduced gender to the binary and did not question power and change (Demetriou, 2001). It was developed at a time where a lot of significant political changes were taking place, especially within the feminist and gay rights movements. In its original formation, and still in some instances, hegemonic masculinity is significantly aligned with criticism of a continuing system of patriarchy. However, Connell and Messerschmidt have argued that the concept has since been oversimplified and partially misunderstood (Connell \& Messerschmidt, 2005; Lomas, 2013). Whilst there is significant concern for the subordination of women, there is also the intermale hierarchy to be considered. The latter is the focus of application in the context of this study. One of the core contributions the concept makes to the study of masculinity is the acknowledgement of multiple masculinities. As hegemonic masculinity forms an inter-male hierarchy there are complicit, marginalised and subordinate forms of masculinity in any given context (Hearn, 2004; Howson, 2006; Donaldson;1993).

Only a small number of men may embody hegemonic masculinity, and thus the values and practices aligned with this most idealised form may not correspond closely to the lives of most men (Connell \& Messeschmidt, 2005). The prevailing values in a broad western 
ideology of masculinity are that of competitiveness, achievement, aggression, and mental and physical toughness (Howson, 2006). How these values are practiced may be different, dependent on the historical, cultural and institutional context given. The variations can be subtle and may require local accounts, such as the specific context of higher education explored within this study. For example, specific localized accounts of hegemonic masculinity have been constructed in places like New Zealand pubs which interweave the concept of dominant masculinity with rural identity (Connel \& Messerschmidt, 2005).

Increasingly the prevailing values associated with hegemonic masculinity in the west, as mentioned above, are being solely associated with the negative characteristics of men (Connell \& Messerschmidt, 2005; Beynon, 2001). This has aligned with the increasing use of the term "toxic masculinity". Hegemonic masculinity has been used to account for violence and crime depicting men as unemotional, aggressive and dispassionate in a blanketing fashion (Connell \& Messerschmidt, 2005). Within the literature on men's health, hegemonic masculinity is often identified as the cause for risk behaviour and the denial of weakness (Lomas, 2013).

The dangers of this trend will be discussed in further detail with regards to masculinity and mental wellbeing. It should be stated at this point, however, that this study is in acknowledgement of the danger of looking at hegemonic masculinity in its negative or constraining forms alone. Hence, the incorporation of enabling factors towards mental wellbeing and the evaluation of both good and bad associated values. As Lomas (2013) discusses, looking at hegemonic masculinity to encompass all the negative characteristics which have resulted in the subordination of women and other men is an oversimplification of the theory. It is entirely possible that a form of hegemonic masculinity may emerge that is almost exclusively positive, and that is something to which we should strive for. Hegemonic masculinity in any context maintains its position through a certain degree of consent and participation, and therefore must too some extent encompass some "benign" or positive characteristics (Lomas, 2013). The focus needs to remain at the localised and individual level, which examines how men individually display masculinity and perhaps why. For this conceptualisation of masculinity at the microsocial level I draw on the various works of Erving Goffman (1959, 1963, 1976). 


\subsubsection{Erving Goffman on gender display}

Erving Goffman's work is extensive, and his theories have been utilised across multiple disciplinary orientations. In his work on Gender Displays (1976, p.69) Goffman states that "if gender be defined as the culturally established correlates of sex (whether in consequence of biology or learning), then gender display refers to conventionalised portrayals of these correlates." This concept has been applied to various studies of masculinity, with it being referred to as a "situational accomplishment" and a "performative act" (Beynon, 2001). Therefore, our focus shifts from what is internal to an individual and takes an interactional and institutional focus (West \& Zimmerman, 1987). It follows on from Goffman's development of the concept of dramaturgical analysis within The Presentation of Self in Everyday Life (1959). Dramaturgy utilises the metaphor of a theatrical performance to describe how individuals present themselves to others. In a sense we are all actors on a stage and our actions or "performances" are determined by the audience that is watching which can be mitigated by specific settings.

The concept of dramaturgy is particularly helpful when exploring masculinity in a regional and institutional setting. Whilst masculinity may be influenced by broad hegemonic values, the setting to which those are enacted is an important factor. For instance, it may be acceptable for a man to exhibit a significant amount of violence when he is partaking in cage fighting, which may be a way for him to enact strength as a value of his masculinity (Giazitzoglu \& Down, 2017). Yet this type of strength may not be able to be displayed in the setting of the man's home or workplace. In a study of boys in London, researchers observed that boys changed between stoic, tough postures and more expressive and emotionally vulnerable stances to align with different social contexts (Oransky \& Marecek, 2009).

West and Zimmermans (1987) concept of "Doing Gender" builds on that of Goffman's Gender Displays (1976). They also contend that the social construction of masculinity is a result of repeated interactions and social processes. However, their work is perhaps further developed towards considerations of gender as a "master status" (Carter \& Fuller, 2015). West \& Zimmerman describe how individuals are consistently assessed on their gender performances across individual and institutional contexts. Therefore "Doing Gender" or displaying gender is unavoidable because this categorization is attached to multiple constructs across social institutions. For instance, when someone is practicing gender in a social situation, gender appropriate or gender inappropriate actions are similarly identifiable. For a basic example, we could look at the prevailing western perspective that a man should 
be mentally and physically strong. This gendered expectation may be practiced in various ways in various situations, but the premise stays the same. West \& Zimmerman (1987) therefore assert that engaging in practices that displaying an individual's masculine values is unavoidable whilst broad ideals of masculinity and the dichotomisation of masculinity and femininity exists.

Goffman's work can be viewed interrelatedly with the concept of hegemonic masculinity. For example, in his work on gender advertisements (1976), he highlights how extensively gender displays are used by advertisers in the corporate realm. Goffman would argue that advertisements do not look strange to us because they reinforce ideals that are already present. However, they should appear strange as they are often overly simplistic and unreachable. West \& Zimmerman (1987) highlight that Goffman views gender as a socially scripted dramatization of cultures idealization of feminine and masculine natures. This is akin to the notion that Connell \& Messerschmidt (2005) present when they discuss the idealised form of masculinity not corresponding closely to the lives of most men. From Goffman's perspective the focus would be on what men would have to do to achieve dominance in the hierarchy. In specific reference to young men in America Goffman (1963, p.128) writes;

In an important sense there is only one complete unblushing male in America: a young, married, white, urban, northern, heterosexual, Protestant, father, of college education, fully employed, of good complexion, weight and height, and a recent record in sports. Any male who fails to qualify in any one of these ways is likely to view himself - during moments at least - as unworthy, incomplete, and inferior.

These expectations and ideals can have potential ripple effects which provide an appropriate segue into a discussion of their impacts on mental wellbeing.

\subsection{Perspectives on mental wellbeing}

Wellbeing, in the broadest sense, is a difficult and elusive concept to define (Dodge et al., 2012; Manwell et al., 2015). In many ways the concept is intertwined with the understanding of values. In fact, the conceptualisation of wellbeing traditionally stems from the philosophy of ethics and how one 'ought to live', which is similar in origin to the study of values (La Placa et al., 2013). An individual's 'Quality of life' has previously been determined by the degree to which their life is 'desirable' as opposed to 'undesirable' (La Placa et al., 2013). Therefore, factors that determine wellness, happiness and a good life are closely aligned with 
cultural values. However, whilst anthropologists have studied values, practices and beliefs across cultures, the consideration of wellbeing is still in its early conceptualisation (Tov \& Diener, 2009).

An important clarification to make when exploring the concept of wellbeing is the distinction between mental illness and mental wellbeing. Historically, there has been a tendency to place these terms on the same continuum (Keyes, 2012; Westerhof \& Keyes, 2010; Slade 2010). However, a new generation of research has surfaced over the last decade that acknowledges individuals may experience diminished mental wellbeing whilst not displaying symptoms of a diagnosable mental illness (Keyes, 2012). As society has progressed, and the treatment of mental illness is more aptly understood, health literature is now looking at wider factors which can influence the wellbeing continuum of individuals and societies. This approach is influenced by the developing field of positive psychology which contests the focus on weaknesses and deficits of traditional psychology. It is concerned with the actions that lead to wellbeing, to thriving communities and various other traits and experiences e.g. optimism, courage, perseverance and love (Seligman \& Csikszentmihalyi, 2014).

Dodge et al., (2012) conducted a review of various proposed descriptions and dimensions for wellbeing to provide a synthesized definition. Their definition of wellbeing involves the use of a "see-saw" as a visual metaphor whereby resources and challenges need to be balanced. In the middle there is the homeostasis of wellbeing. Therefore, an individual or society should maintain a balance of resources in relation to challenges to facilitate the "homeostasis" of wellbeing. This study incorporates a similar notion of a "see-saw" by exploring both enabling and constraining factors that are associated with the nexus of masculinity and mental wellbeing.

\subsubsection{Concern for the mental wellbeing of men}

Concern for the mental wellbeing of men is increasing both internationally and in New Zealand (Pitman et al., 2012; Mckenzie, 2017). Since the 1970's there has been a significant rise in suicide rates for young men in OECD countries (Pitman, 2012). Concurrently however, women have been diagnosed with depression at higher rates than men. Due to the high comorbidity rate between depression and suicide there is a contradictory pattern presenting itself, which suggests there is perhaps a disparity in behaviours surrounding mental health enactment and help seeking. We know that men are more likely to be diagnosed with conduct disorders and act out aggressively (Lomas, 2013). However, reasoning for these 
disparities in mental illness rates are poorly understood (McKenzie, 2017). Within the mental health literature and diagnostic criteria, the consideration of the social nature of gender is often not considered in-depth.

In a study by Brownhill et al. (2005) the term "the big build" was used to describe a behavioural pattern amongst men whereby emotional distress is suppressed and later expressed in the forms of aggression or violence. Whilst the self-reported experience of emotions showed little difference between males and females it was the outward expression that highlighted a contrast. Another example of a social behaviour towards health practices is the condonement of risk-taking and toughness that is celebrated amongst men at higher rates than women. Courtenay (2000) describes how when a man positively states that he "hasn't been to a doctor in years", he is simultaneously describing a health practice and displaying his masculinity.

\subsubsection{Erving Goffman on stigma}

The differences in the display and attitudes towards both mental wellbeing and mental illness are largely influenced by the values commonly associated with masculinity. For example, the lack of help-seeking by men has been tied to values such as self-sufficiency and selfcontrol (Johnston et al., 2008). Erving Goffman's work on stigma (1963) provides a useful theoretical and sociological perspective on this pattern of behaviour. When discussing stigma, Goffman (1963) explains that society establishes the means of categorising persons. Alongside that category are attributes which are felt to be ordinary and natural for members of that category (Goffman, 1963). When someone displays a value or practice that sets them apart from persons in their category, this is a stigma.

The term originated from the Greeks and referred to bodily markers such as cuts or burns that would indicate an individual was a slave, criminal or traitor. In contemporary use it is more broadly applied in a sense that any unanticipated difference can result in a stigmatised individual. A stigmatised individual is often met with adverse reactions from wider society. Goffman (1963) describes how we tend to use demeaning terms such as "cripple" or "moron" without regard for their original meaning and overcompensate in our attributions of criticisms based on a singular "defect". As a stigma is often directed towards that which deviates from the average, fear of the unknown can cause people to react disproportionately.

Being stigmatised can cause detrimental effects to a person's mental wellbeing for multiple reasons. The fear of being stigmatised and segregated can be restrictive, causing people to 
forgo their needs and wants. Then the adverse reactions that are received when one is stigmatised can cause many negative feelings such as shame, embarrassment, hurt and loneliness. Until recently, a very unfortunate pattern has existed whereby mental illness was deeply stigmatized in many societies across the world. Goffman (1963) viewed mental illness as one of the most damaging stigmas to have existed. Socially isolating those who are already fighting internal battles only results in the compounding of suffering. Finzen (2017) has described this pattern as a "second illness".

\subsubsection{The crisis of masculinity and a dual stigma}

Perceptions of masculine ideals adds yet a "third layer" for men dealing with mental illness or diminished mental wellbeing. The idea that males are more competitive, aggressive and less emotional than women is a prevailing ideology in many cultures. However, this notion is becoming increasingly scrutinized, resulting in the use of the phrase "crisis of masculinity". This phrase can be frequently found across the literature on men (Sim \& Thomson, 2000; Gee \& Jackson, 2012; Itulua-Abumere, 2013). At the outset of this thesis a quote by Thomas Carlyle in 1831 was presented (Adams, 2018, p.1). This statement effectively highlights that the concept of masculinity "in crisis" is not a new one; however, it is an elusive concept and particularly difficult to define (Itulua-Abumere, 2013).

As "traditional" values and traits associated with masculinity become less applicable in society, new conceptualisations go through a struggle towards becoming concrete (Gee \& Jackson, 2012). Beynon (2001) argues that because masculinity is fluid and in constant reform, it is unclear whether masculinity is constantly in crisis in some shape or form. In 1998 Hopkins and Riley (as cited in Sim \& Thomson, 2000) discussed this concept in specific reference to the "kiwi bloke":

For the confused regiments of modern-day blokes who never consciously patriarchally oppressed anybody, the condemnation has all been rather disorientating and disheartening.... We've had to come to terms with being emotionally cold, excessively angry, unhealthily sexist and rather prone to dying sooner than we should. We're the new noxious pests - an endangering species even. Poised on the cusp of the millennium, masculinity is a downright liability.

Although this was written twenty-one years ago, it applies to the term "toxic masculinity" which, as previously discussed, has increased in usage in recent years. Therefore, there is a potential for a "dual stigma" because whilst traditional masculine values are becoming increasingly scrutinized there are still areas of society, particularly male-dominated areas, 
where acting against those values or practices is also stigmatised. Robertson (2007) describes it as a "double whammy" and highlights that men are discursively situated as both "risk takers" and those "at risk". Lomas (2013) also describes the same notion and discusses how both in academic literature and society men are positioned as "damaged and damage doing". As stated, stigmatization is commonly reinforced within male dominated areas or within male friendship groups. Whilst strong male friendships and brotherhood can foster positive social relationships and facilitate wellbeing, there are many instances where large male groups can induce negative values and practices (Oransky \& Marecek, 2009). This can begin at a young age. William Pollock (1999), author of "Real Boys: Rescuing Our Sons from the Myths of Boyhood", describes the emotional isolation and emotional illiteracy that is impinged on boys via a stringent "boy code". The idea of "no sissy stuff" is discussed in detail, with intimate friendships between boys being negate of dependence, warmth and empathy.

\subsubsection{A focus on positive forms of masculinity}

Part of the concept of multiple masculinities means also acknowledging that men can strategically negotiate the values and practices associated with hegemonic masculinity. Therefore, they can simultaneously endorse and challenge traditional expectations of masculinity (Lomas, 2012). This could mean being in complete opposition or reinventing the same values through different practices. In a study by Harris (2010) college students were interviewed to discover their "meaning of masculinities". It was found that being "confident and self-assured" was associated with masculinity. However, the students reported that this "masculine concept" could be used to reject masculine norms and make conscious decisions to perform masculinities in ways they deemed appropriate. They reported that exuding selfassurance in their decisions, which were contradictory to masculine norms, meant they were able to do so without being questioned. This open rejection of masculine norms was similarly found in a study by Hong (2000). This study examined behavioural and attitudinal changes among eight men who participated in a campus violence prevention program. The men openly rejected masculine norms and prioritized empathy, service, leadership and nonviolence.

Despite studies which highlight the variation of attitudes towards reframing traditional masculine norms, there is a gap in the literature that addresses positive forms of masculinity (Englar-Carlson \& Kiselica, 2013; Lomas et al., 2016). The practices that men may 
traditionally associate with masculinity, mainly physical endeavours, can also be used to facilitate a positive sense of both physical and mental wellbeing. For instance, sport can be used as a healthy medium to facilitate the comradery, courage and leadership values traditionally and positively associated with masculinity. A good example of this is the Australian not-for-profit organization "Men's Sheds", which brings men together to work on physical projects in order to alleviate feelings of loneliness (Woosler, 2018). Exploring ways in which men can or do act in ways that has a positive impact on their mental wellbeing is important in instigating widespread change.

A study by Lomas et al. (2016) is one of the few recent studies that specifically looks at masculinity and the facilitation of positive mental wellbeing. Within the research Lomas et al. (2016) describe how positive hegemonic norms that are conducive to mental wellbeing can arise in "communities of practice" or specific localised contexts. They describe how in 2009, Duncanson found a specific "peacekeeper masculinity" emerging within a military context and challenged the hegemonic "warrior masculinity". Soldier's mental wellbeing was being facilitated through the new-found ability to bond with local communities, which removed hostility and isolation. However, as a result of their study, Lomas et al. (2016) found that taking on "new ways of being a man" in localised contexts was frequently challenged due to pressures to conform to new practices whilst simultaneously being pressured not to adopt these practices by external communities of men.

\subsubsection{Mental wellbeing of male students in higher education}

The mental health and wellbeing of higher education students has been of critical international concern in recent years (Baik et al., 2019; Lipson \& Eisenberg, 2018). A growing number of empirical studies are indicating that university students are a high-risk population for psychological distress and mental health concerns (Baik et al., 2019). It has been suggested that this may be due to that the typical age of university students (18-24 years) coincides with the typical age of onset for many mental illnesses (Lipson \& Eisenberg, 2018). However, as discussed earlier in the review, mental wellbeing is not just the presence of mental illness. In terms of higher education this means that looking at the prevalence of mental illness rates is only one means of assessing the mental wellbeing of students. The transition from home and family life can be a significant stressor, as well as having first time financial worries and being newly responsible for most of your own learning. Drop-out rates and campus misconduct are two other possible indicators of diminished wellbeing. 
Most students that commit non-academic violations on American college campuses are men (Harris, 2010). There has also been a significant focus within the literature on reports of academic underachievement, alcohol and substance abuse, homophobia, and poor coping amongst college men (Harris, 2010). Most notably the underachievement of men in higher education is becoming an increasingly recognised phenomenon. Across all levels of education, it is becoming apparent that boys and men are underachieving in comparison to females. There is no explanation as to the cause of this pattern, only theoretical proposals. It was only about ten years ago when scholars began to explore the relationship between issues that challenge college men and the social construction of masculinity (Harris, 2008).

\subsection{The cultural context: New Zealand}

This study is based in New Zealand, a geographically remote country in the south pacific consisting of two main islands, with a population of approximately 4.5 million (Kennedy, 2007). Māori are the indigenous people of New Zealand, who have a unique culture and traditions. In 1841 the country was colonised by Britain and there was a large increase in European settlers (King, 2003). Over time the link to European heritage faded for the settler population. A sense of cultural identity has developed, with most of the New Zealand population describing themselves as "New Zealanders" or "Kiwi" (Kennedy, 2007). The dominant form of masculinity in New Zealand is the traditional "kiwi bloke" or "Southern man" and stems from the colonial context. As previously stated, the theoretical underpinning of this research surrounds the enactment of masculinity, and therefore acknowledges the multiplicities of masculinity in New Zealand. However, it is important to gain and understanding of the most dominant or idealised form of masculinity in order to establish a frame of reference for how other masculinities may be positioned.

\subsubsection{The "kiwi bloke"}

The traditional "kiwi bloke" or "southern man" has been the dominant identifiable masculine archetype in New Zealand for some time (Sim \& Thomson, 2000; Joseph \& Falcous, 2017). It is also a marker of New Zealand culture in general. For example, in a 2004 study on national identities of the Unites States, Australia, and New Zealand, researchers noted the continuing "blokiness" of New Zealand culture (Bannister, 2005). The symbols identified by both males and females that represented New Zealand culture were all similar i.e. rugby, gumboots and tractors. For the US, the symbols tended to be more gendered with distinct symbols representing both American women and men. As discussed above, the idea of a dominant masculinity or hegemonic masculinity acknowledges that there will be 
marginalized or subordinate masculinities present. Hegemonic masculinity is a complex concept that presents itself differently in different contexts and cultures with the subordinate and marginalized groups often overlapping and integrating with the dominant archetype in different ways. For example, Bannister (2005) highlights that the kiwi bloke in a UK context would be marginalized because of his stereotypical proletarian class identity. Therefore, the kiwi bloke would not be a dominant archetype for UK national identity as it is in a New Zealand context.

Prior to the $21_{\text {st }}$ century the kiwi bloke phenomenon was not discussed extensively within academic literature (Sim \& Thomson, 2000). The literature that includes a discussion of the "kiwi bloke" is predominantly concerned with sport (Joseph \& Falcous, 2017) and drinking (Willot \& Lyons, 2012), which reflects the prevalence of these practices to this form of masculinity. Sim and Thomson (2000) credit Jock Phillips with providing the most detailed account into the historical development of Pākehā male identity in New Zealand. Pākehā is the Māori term that refers to white settlers in New Zealand, and whilst not all Caucasian New Zealanders identify with the term, it is used repeatedly in historical accounts of the development of masculinity in New Zealand (Bannister, 2005). I separate this discussion into two parts. The first is concerned with the wider values associated with the "kiwi bloke" and the second surrounding common practices.

Phillips identifies the early pioneering lifestyle, colonialism, two world wars, rural labor and rugby as crucial to the origin of the Kiwi bloke (Sim \& Thomson, 2000). It is this historical context which has installed some of the common values that characterize a national-level hegemonic masculinity in New Zealand. Earlier, reference was made to some common western values associated with hegemonic masculinity e.g. competitiveness, achievement, aggression and mental and physical toughness. There are similarities and influences between these values and those which influence the ideology of the "kiwi bloke". However, perhaps due to the influence of the rural landscape, pioneering lifestyle and physical representations of masculinity, the "kiwi bloke" places significant emphasis on physical and mental toughness. In a historical sense, the culture has been described as one "of the body rather than the mind" (Bannister, 2005, p.2).

There is also a significant emphasis placed on being "one of the boys", perhaps formulated by that early mate ship and comradery when faced with strenuous physical tasks. Sim \& Thomson (2000, p.4) describe the kiwi bloke and associated values in a succinct way; 
"Men are characteristically loyal to their mates, constantly exchanging banter...but most importantly displaying masculine feats of strength, courage, competency with machinery, and denial of pain. There is always a constant undermining of emotions".

This undermining of emotions also falls into the historically valued trait of modesty, with New Zealand men pointedly expressing dislike for those who are "too big for their boots" even if in positive expression of their own achievements (Sim \& Thomson, 2000, p.11).

Sport, and most specifically rugby union, has historically and continually been a very significant part of practicing dominant masculinity in New Zealand (Joseph \& Falcous, 2017). In an early, colonial society, rugby provided New Zealanders with national integration and something common to share (Sim \& Thomson, 2000). It therefore became a way to simultaneously solidify male identity in New Zealand but also the establishment of the colony as a country. Sport, in general, has been observed as a significant site for constructing and practicing dominant masculinities (Tagg, 2008). Not only does it consolidate physical prowess, mate-ship, and tolerance of pain but the specific sport can be a symbol of masculinity. For example, men who play netball in New Zealand are typically stereotyped as gay and mocked by males who conform to dominant masculinity (Joseph \& Falcous). Also, historically, men refusing to wear protective equipment in sport was commonplace and there was admiration for those who could ignore their injuries (Sim \& Thomson, 2000).

Like sport, drinking, is also something that has been a significant part of dominant male identity in New Zealand since colonization (Willot \& Lyons, 2012). Beer is often a symbol of an individual's entry into manhood both in New Zealand and other western societies (Willot \& Lyons, 2012). To quote a participant in a study conducted by Penwarden (2010, p.47) "That's part of being a man. It's being able to handle your piss". Also, from a novelist depicting Spanish Machoism (Courtenay, 2000 p.1390);

A macho doesn't show weakness. Grit your teeth, take the pain, bear it alone. Be tough. You feel like letting it out? Well then let's get drunk with our compadres... Drinking buddies who have a contest to see who can consume the most beer, or the most shots of tequila, are trying to prove their maleness.

Dependent on geographical location, beer and the advertising of beer has often portrayed idealized forms of masculinity. Most significantly, for New Zealand, this is evident in the Speights beer “Southern Man' advertising campaign (Gee \& Jackson, 2012; Willot \& Lyons, 2012). The campaign "mobilizes imagery of the South Island High Country to present a 
particular construction of masculine identity" (Law, 1997). There are also a host of other beer advertising campaigns in New Zealand that have sought to reflect the dominant ideal of a kiwi man, such as Lion Red's The measure of a man's thirst advertising campaign and the DB Patron Saints of Beer Drinkers advert (Penwarden, 2010).

Due to the context of this study, it is also appropriate to highlight the relation between the "kiwi bloke" and higher education. Bannister (2005) discusses how, historically, the "kiwi bloke" raised questions about the masculinity of intellectuals. Intellectuals and most pointedly, writers, felt a pressure to emulate dominant masculinity, proving their solidarity with "ordinary blokes". Specifically, this is done through the practice of heavy drinking, as displaying masculinity through physicality in things such as sport was not as much of an option. Whilst going to university is now very commonplace for both men and women, it is possible that there is still a similar pressure that exists for men attending university. Willot and Lyons (2012, p.332) discuss how men at university in America have been found to use public drinking to demonstrate their "hegemonic status to others".

\subsubsection{The mental wellbeing of New Zealand men}

Farming, rural isolation, an emphasis on physicality and a national drinking culture are all social and historical factors that can influence attitudes and behaviors surrounding mental wellbeing. Whilst self-sufficiency and self-control are values that are associated with broad western conceptualizations of masculinity (Johnston et.al., 2008), New Zealand has a unique perspective on these values. Most New Zealanders are familiar with the "harden up" dialogue which entails "getting on with it" without complaint or discussion.

Over the past year, the mental wellbeing of New Zealand men has been highlighted extensively in the media (Kightley, 2018; Skerrett, 2019; Marvelly, 2019). There are now multiple movements and programs that seek to address this issue from different perspectives, and that mostly center around encouraging men to open up and ask for help. For instance, "Farmstrong" is a nationwide wellbeing program for the rural sector (Skerrett, 2019). It aims to encourage farmers to engage in discussion surrounding mental wellbeing to target the number of farmers taking their own lives. New Zealand's national rugby team, the All Blacks, has also set up a "Vulnerability group" to create a safe space for players to share issues and support one another (Marvelly, 2019).

However, academic research into men's mental wellbeing in New Zealand is limited. Mckenzie (2017) is one of the few researchers in New Zealand that has explored men's lived 
experiences and how this effects mental wellbeing. Her research uses a gender-relations approach and life history method, which explores the life of men aged between twenty and forty years of age. This study differentiates by focusing on the organizational context of higher education institutions in New Zealand.

\subsubsection{Mental wellbeing and male university students in New Zealand}

In New Zealand, the age cohort with the highest number of suicides for the 2017/18 period was the 20-24-year-old group (Ministry of Justice, 2018). The majority of New Zealanders within that age group attend tertiary institutions across the country. This makes universities an appropriate and perhaps integral site for the exploration of this age group and their wellbeing.

Recently, tertiary students in New Zealand have voiced the need for more mental health support in universities (Long, 2018). The premise is that, attending university can often lead to feelings of being overwhelmed and fear of failure, which in turn can cause underlying mental health issues to surface ("Tertiary Student Leaders", 2018). The president of the New Zealand Union of Students Association, Johnathan Gee, recently commented on the complex nature of the university as a working organization ("Tertiary Student Leaders", 2018):

"A university is the size of - and has many of the issues of - a small town, including issues of mental distress."

This statement highlights that although higher education is a specific organizational context, it is a very diverse and complex environment. To support students, it is therefore appropriate to explore specific demographics, such as men, and the ways in which their experience of university may be unique. There is a significant gap in the literature that explores the experiences and mental wellbeing of male students in New Zealand. Otago university recently reported that women outnumbered men on university campuses at a ratio of 60:40, with similar statistics being shown across all universities in New Zealand (McPhee, 2019). The reason for this gap is largely unknown. Personal stigma surrounding mental health is also significantly evident amongst male university students (Eisenberg et al, 2009), therefore, understanding the ways in which masculinity shapes views on mental health within this context may be particularly imperative. 


\section{Chapter 3}

\section{Methodology}

This chapter outlines the research process and is divided into three sections. The first explains the disciplinary and theoretical areas that informed this research. The second section details how the data was collected. Finally, the third section explains the data analysis and categorisation process.

\subsection{Informing the methodological approach}

This study was conducted using an exploratory approach. The topic leant itself to qualitative research, which allowed for the in-depth exploration of the subjective experiences and perspectives of the participants. The study was inductive, and seeks to develop concepts and understandings, rather than confirming preconceived hypotheses (Taylor et al, 2015). It maintained a focus on perceived cultural values and practices. This section begins with a discussion of the disciplinary orientation to which this study is situated. It then explains the theoretical perspectives that informed this study. The works of R.W Connell (Connell, 1985, 1997, 2005; Connell \& Messerschmidt, 2005) and Erving Goffman (1976, 1963, 1959) have been discussed in relation to masculinity and mental wellbeing, in this section their work will be discussed in relation to the methodological approach. The section concludes by outlining the formation of the interview guide.

\subsubsection{Disciplinary orientation: Cultural studies}

This study is situated within a cultural studies approach. There is significant variability in the way in which the term "culture" is used (Schein, 2010; Alvesson, 2012). Predating the mid nineteenth century, culture had two very different contextual applications. The first of those applications was the use of culture to refer to a differentiation between "high" and "low" class systems, such as "he was very cultured" (Morgan, 2006). This application is now rarely used in contemporary society. This is in part due to the early formation of British culture studies as a discipline. Raymond Williams, one of the founders of cultural studies, sought to valorise the potential of working-class culture, and remove the distinction between "high" and "low" culture imposed by the upper class (McGuigan, 1997). Whilst the basic differentiation between "high" and "low" culture is no longer predominantly utilised, tension between cultures is something that has remained a focus in cultural analysis. For instance, 
culture studies began to focus on the idea of oppositional subcultures to that of dominant or mass culture.

The second application, and the one most currently referred to, is a broader conceptualisation of culture which originates from anthropology. This conceptualisation of culture is perhaps less concerned with the cultural tension previously described, and instead surrounds what comprises a specific culture. Drawing on Gareth Morgan's (2006, p.116) description, culture is “the pattern of development reflected in a society's system of knowledge, ideology, values, laws and day to day ritual." However, as previously stated, culture can vary considerably in it use as a term and concept. In 1952 Kroeber \& Kluckhohn critically reviewed the concept of culture and compiled a list of 164 definitions. This effort to define culture continues, and as of 1993 Sheryl Lindsley and John Baldwin published a compilation of 200 definitions (Baldwin et al., 2006). It is also now recognised that culture can operate at various levels. For instance, many people recognise differences in national culture, however there are also regional, generational and corporate or organizational cultures to consider. It is therefore necessary, when undertaking a cultural methodology, to describe the specific elements of culture that will be used for cultural identification (Alvesson, 2012).

In this study the core focus is on perceived cultural values and practices. Edgar Schein (2010) proposes that there are three levels of organizational culture, which can also be applied to culture more broadly. The first level is artefacts, which are the more easily identifiable aspects of culture, for instance; clothing, language, rituals and practices. Practices are deemed cultural when there is a shared perception of how people behave within a culture (Frese, 2015). Whilst practices may be easy to describe and observe, we cannot attribute meaning to practices without understanding why they are taking place. Therefore, it is also necessary to explore values.

The second level to Schein's framework includes values or beliefs. Whilst they are not as easy to identify they can still be accessed through participants acclamations of what they value or perhaps what they perceive others to value. Values, in this context, allow for the differentiation between what is "good" and what is "bad". In his description of Kluckhohn's definition of values, David Graeber (2001) highlights the term "desirable". He makes a very important distinction; individually people desire many things, but values are ideas surrounding what people ought to desire. Values act as a guide for individuals to determine what desires they should act upon and which ones to ignore. 
The third level to culture, underlying assumptions, are often unconscious and unidentifiable. Given the methodology, this studies focus is to identify cultural values and practices. It stops short of attempting to explore psychological roots.

\subsubsection{Theoretical perspectives of Erving Goffman and R.W Connell}

This research is informed by a social constructionist epistemology. The primary underlying assertion of a constructionist approach is that knowledge is formulated through social relationships as opposed to individual an internalised rationale or motivation (Gergen, 2015). Consistent with this approach, an interdisciplinary conceptual framework of theoretical perspectives is used.

R.W Connell and Erving Goffman share the theoretical perspective that masculinity is performed (Hein \& O'Donohoe, 2014). Connell's work has previously been drawn upon to explore masculinity through a social constructionist epistemology (Harris, 2010; Barron, 2009). This perspective is focused on the concept of masculinity as a product of social interaction rather than being inherently or intrinsically part of an individual. The theoretical perspectives of Goffman and Connell imply that researchers are able to "investigate how masculinities are done and how these performances are received within social interaction; how frames, schedules, and specificities of culture and history condition masculine performances and their reception; how tensions around front- and backstage play out; and how illusions of masculine authenticity are reproduced and congealed" (Brickell, 2005, p.32).

\subsubsection{Formation of the interview guide}

Data collection for this research utilised semi-structured interviews, which are particularly well suited when it comes to exploring participants' perceptions of complex and possibly sensitive topics (Barribal \& While, 1994). The interview guide (see Appendix A) ensured that the interviews explored the relevant and consistent constructs. However, the semistructured process enables one to gain clarity and depth with regards to any answers given (Barribal \& While, 1994). The explorative nature of this study meant it was necessary to be able to ask "follow-up" questions. This also served to create more genuine rapport between the researcher and participant.

Firstly, introductory questions were used to make participants comfortable. They were also used to establish participants' stage of study and whether they had lived in New Zealand for most of their lives. The second stage of the interview questions were designed to explore 
perceptions of masculinity. Participants were asked how they would describe an "average kiwi man". The question "who do you think might embody or portray this type of man?" was then used as a prompt if participants were having difficulty conceptualising this answer. It was also an effective way to generate more depth of discussion.

The interview guide then placed emphasis on the practices of the "average kiwi man". Therefore, participants were asked "what does this man do?". Alternative descriptive terms that were used throughout the interview process were "social practices", "activities" or "things that people do socially". Subsequently the exploration of values was incorporated by asking participants "what do people think is important to the average kiwi man?" and by using the following prompts; "values", "morals", "care about". Throughout this section participants were also asked whether there was a general expectation that men act in accordance with these values and practices. This question allowed for a link to be made between possible stereotypes participants were describing and their perception of men's lived experiences.

The final section of the interviews was used to tie together what had previously been discussed to the concept of mental wellbeing. Here the effects on wellbeing were explored. Participants were asked how the practices and values that they identified could be good for (enable) or bad for (constrain) mental wellbeing. The final questions addressed whether the participants perceived being a male student in New Zealand affected mental wellbeing.

\subsection{Data Collection}

This section describes how this research was conducted. It addresses the process of recruitment and conducting the interviews, a discussion surrounding the participants as well as the positionality of the researcher.

\subsubsection{Conducting the interviews}

Prior to conducting the interviews, ethics approval was granted by Victoria University of Wellington's Pipitea Human Ethics Committee. Participants for this research were recruited via a mixture of "snowball sampling" and "volunteer sampling". First an advertisement was created which highlighted the requirements for participation in this study (see Appendix B). The requirements were that the participant must identify as male, between the ages of 18-25 and that they must currently be enrolled at a tertiary institute in New Zealand. For the snowball sampling method, this advertisement accompanied by a participant information sheet (see Appendix C) was given to acquaintances and university staff. This meant that 
participants could then be referred to me. The advertisement was also placed around university campuses in the Wellington region in order to recruit volunteers. A participant draw was added (whereby each participant would go in to win a $\$ 50$ supermarket voucher) to provide incentive for the students to offer their time for this study.

When a participant volunteered or was referred, I contacted them via email to answer any further questions and arrange a time for them to be interviewed. Each interview was conducted on the preferred campus chosen by participants. A consent form and information sheet (see Appendix D) was provided to participants prior to conducting the interview. They were given time to read through both forms and were asked if they had any further questions. With the participant's consent, each interview was audio recorded and lasted up to an hour. Throughout the interviews, I took notes of any novel or unexpected answers and discussions so that they could be built upon through follow up questions. This was important for reflexivity and to make sure that follow-up questions were not only orientated around preconceived conceptions.

\subsubsection{Participants}

Six postgraduate and six undergraduate students participated in this study. Although this research did not aim to gather a representative sample of both undergraduate and postgraduate, I endeavored to make sure participants came from a range of different stages of study. All the participants were born in and grew up in New Zealand. They were also studying in a range of different disciplines from design through to geography and business. It is not possible to know whether specific academic disciplines significantly influence the perspectives and answers provided by participants. However, in one case insights and perspectives that were provided by a participant were perhaps influenced by his previous studies of gender:

"I'm trying to resist the urge not to go into feminist theory here"

There was another participant who had volunteered in student wellbeing and provided insight from this area. Other participants also incorporated perspectives from their respective disciplines, for example, economics and statistics or student stress in the design school specifically. The research did not intend to gather a representative or comparable sample of participants from all disciplines. However, it was beneficial to have participants from different subject areas in order to broaden the insights and perspectives. 
Recruitment stopped after twelve participants had been interviewed. The number of participants required within a qualitative study is debated within the literature. It can vary based on the type of methodology utilised and the way in which a study is designed. One of the primary methods used to justify the number of interviews within a qualitative study is thematic saturation. Research by Guest, Bunce \& Johnson (2006) is one of the first academic papers that systematically documented a process of reaching saturation by twelve interviews. Ando, Cousins \& Young (2014) also found that during their analysis of saturation all themes and codes were solidified by twelve interviews. This current study did not specifically restrict itself to twelve interviews, however, after eight interviews the themes identified did not change. The four subsequent interviews added further depth to the overall data set.

\subsubsection{Positionality}

Qualitative research has two sides: that of the researcher and that of the participant (Bourke, 2014). Therefore, it is impossible to ignore the interpretations and standpoint of the researcher. I was consistently aware of my position as a female of somewhat similar age to the participants. The gender of the researcher is particularly important to consider when the topic is exploring a gendered concept such as masculinity. It is possible that being a female researcher could have influenced the way in which the male participants responded. Schwalbe and Wolkomir (2006) wrote an extensive guide on possible issues with the "masculine self" and interviewing men. They draw on Goffman's (1959) dramaturgical approach to argue that an interview scenario may be a situation that involves a performance of masculinity.

Therefore, it is important for the researcher to note their part in this performance. They highlight that interviews regarding the concept of gender has the potential to be perceived as threatening by male participants. They state that this "threat" can be heightened when the researcher is female. However, Bridges (2013) suggests adopting the "least masculine role" when interviewing men to minimise inexpressiveness. For instance, a male participant may not want to discuss a sensitive topic in-depth with someone they perceive to be overtly dominant and masculine.

It is not possible in this case to be fully aware of how being a female researcher may have affected the data that was collected for this study. A comfortable rapport was established with all participants, and on a few occasions, there were in-depth discussions surrounding personal experiences. Overall, I did not perceive any issues with the level of engagement and 
expressiveness throughout the interviews. There were various instances where participants would use statements such as "you know" (how it is). It is unclear whether this was because of my similar age and status as a student, whether they believed what they were discussing was common-sensical, or whether it was to avoid going into further depth on a question. Schwalbe and Wolkomir (2006) discuss how handling these types of statements needs a level of balance from the researcher. This is because the participants could be using them to establish a shared bond or level of understanding but if statements like these are used too often it can lead to too much interpretation required by the researcher. To overcome these instances, I would use phrases such as "I think I understand what you mean, but could you explain anyway"

\subsection{Data Analysis}

This section details the method used to analyse the data. After each interview, the audio recording was used to transcribe the interviews verbatim. Pseudonyms were used for the transcripts to ensure confidentiality. During the first four interviews I began analysing potential themes and coding them by hand. However, I subsequently began entering the transcripts into the coding program NVivo 12. This allowed me to more effectively add additional codes and return to the data that was under each individual theme. Initially the answers that were given by participants within each section of the interview (description of an "average kiwi man", practices, values and mental wellbeing) were organised under the interview sections comprising these four main themes. These overarching themes stayed consistent throughout the analysis process with the sub themes within them altering and/or merging. These alteration or merges took place over the course of the interviews and during the final stages of analysis. The process of forming categories required the transcripts to be read repeatedly alongside listening to the audio recordings.

An adapted version of Mark Constas' (1992) two-dimensional model of category documentation was used (Table 1). The table presented only includes the elements relevant to this current study. The model provides a way to effectively communicate the categorization process. The first domain is the Component of Categorization and it identifies actions associated with the development of a category. The second domain is Temporal Designation which indicates the stage that a category was developed within the study. Categories may be created a priori (or before the data is collected), a posteriori (or after the data was collected), or iteratively (at various points throughout the data collection process). 
Table 1

Adapted version of Constas' (1992) two-dimensional model of category documentation

\begin{tabular}{|c|c|c|c|}
\hline \multirow{2}{*}{$\begin{array}{l}\text { Component of } \\
\text { Categorization } \\
\text { Origination }\end{array}$} & \multicolumn{3}{|c|}{ Temporal Designation } \\
\hline & A priori & A posteriori & Iterative \\
\hline \multicolumn{4}{|c|}{$\begin{array}{l}\text { Where does the authority for } \\
\text { creating categories reside? }\end{array}$} \\
\hline - Participants & & & $\begin{array}{l}(1 b)(3 a)(3 b)(2 a) \\
(2 b)(4 a)(4 b)(4 c)(4 d)\end{array}$ \\
\hline - $\quad$ Literature & $(1)(2)(3)(4)$ & & $(2 a)(2 b)(4 d)(1 c)$ \\
\hline - Interpretative & $(1)(2)(3)(4)$ & (1a) & $(3 a)(3 b)$ \\
\hline \multicolumn{4}{|l|}{ Verification } \\
\hline \multicolumn{4}{|c|}{$\begin{array}{l}\text { On what grounds can one } \\
\text { justify a given category? }\end{array}$} \\
\hline - Rational & & $(3 a)(3 b)$ & $(2 a)(2 b)(4 d)$ \\
\hline - $\quad$ Referential & & & $(1)(2)(3)(4)(1 c)$ \\
\hline - Empirical & & (1a) & $(1 b)(1 c)(4 a)(4 b)(4 c)$ \\
\hline \multicolumn{4}{|c|}{ Nomination } \\
\hline \multicolumn{4}{|c|}{$\begin{array}{l}\text { What is the source of the } \\
\text { name used to describe a } \\
\text { category? }\end{array}$} \\
\hline - Participants & & & $(4 a)(3 b)(4 b)(4 c)$ \\
\hline - Investigative & & & \\
\hline - $\quad$ Literature & & & \\
\hline - Interpretative & $(2)(3)(4)$ & $(1)(1 a)(1 b)(3 a)(1 c)$ & $(2 a)(2 b)(4 d)$ \\
\hline
\end{tabular}

\begin{tabular}{|c|l|}
\hline Category Label Key: & \\
\hline (1) The "average kiwi man" & $\begin{array}{l}\text { (1a) Generational difference } \\
\text { (1b) Richie McCaw and the All Blacks } \\
\text { (1c) The student }\end{array}$ \\
\hline (2) Cultural Practices & $\begin{array}{l}\text { (2a) Physicality } \\
\text { (2b) Drinking }\end{array}$ \\
\hline (3) Values & $\begin{array}{l}\text { (3a) Family values vs values of emerging } \\
\text { adulthood } \\
\text { (3b) Loyalty between men vs self-reliance }\end{array}$ \\
\hline (4) Mental Wellbeing & $\begin{array}{l}\text { (4a) Social bonding vs social ostracism } \\
\text { (4b) Banter } \\
\end{array}$ \\
& $\begin{array}{l}\text { (4c) Competition } \\
\text { (4d) Devaluation of emotional expression }\end{array}$ \\
\hline
\end{tabular}

\subsubsection{Origination}

The three authorities for creating categories stemmed from the participants, literature and interpretation. Sometimes the categories were created via a mixture of the various elements within the table. For instance, the four main themes were generated before data analysis or 
"a priori" as they were initially used to organise the data and they also stemmed from the interview guide which was structured around relevant literature. Subsequently an iterative process was used to verify these four key themes. This involved questioning their necessity in representing the data. Whilst the generation of the sub themes ultimately came from various points throughout the interviews, the organization of the subthemes under these four key themes remained appropriate. The naming of these categories remained the same except for "the average kiwi man".

Constas (1992) notes that the iterative temporal designation is most commonly used, which was found to be the case during this study. In the iterative temporal designation, categories may be created at various points during the research process. This aligns with qualitative research being a reflexive and evolving method of research. Most of the categories in this study originated from the participants discussions but were created iteratively. For example, the category "Richie McCaw and the All Blacks" originated from the participants, however, its substantiation as an independent category formed over time as different levels of emphasis were made by participants. The categories "physicality" and "drinking" originated from a mixture of the academic literature and the participants. The literature that was gathered for this research indicated that physicality and drinking were prominent signifiers of masculinity in New Zealand. However, these two practices were also overwhelmingly discussed by participants.

\subsubsection{Verification}

There were three grounds for verification in this study. The first, 'rational' verification, relies on logic and reasoning to verify the categories. This is relevant to drawing connections between findings to create categories through the interpretative approach. For example, the sub-theme "Loyalty between men vs self-reliance" was created a posteriori from participants insights and it was previously two separate sub-categories. After consideration, I found that the categories had comparative and contrasting elements and so were more suitable together.

'Referential' verification involved using existing theoretical components and research findings. This was the case for the four main themes. 'Empirical' verification of categories stems from data that warrants a category without reference to other studies. Most of the categories under the theme "Mental Wellbeing" are included here. For instance, the impact of competition on mental wellbeing was not something that was represented in the gathered literature and was discussed extensively by participants. The category, "The student", 
originated from a consideration of the interaction between student identity and "the average kiwi man" from the literature.

\subsubsection{Nomination}

The final component of categorization is 'nomination' which is concerned with the naming of categories. There were two sources for nomination within this study, participants and interpretation. For example, the term "competition" was explicitly used by participants. Whereas "physicality" was a category descriptor that went through various stages of development due the various types of emphasis that was placed on different physical practices. The holistic emphasis placed on physical activity, including sport, warranted a broader category name.

\subsubsection{In closing}

To conclude, the research design of the study is exploratory and qualitative, underpinned by a culture studies approach. It does not seek to provide definitive or generalisable conclusions. It does seek to provide insight on perceptions of masculinity and mental wellbeing within a unique cultural context. The next chapter provides an expanded discussion of the themes identified here. 


\section{Chapter 4}

\section{Findings}

This chapter presents the findings that arose from the twelve interviews and subsequent data analysis. Four key themes were identified; the "average kiwi man", cultural practices, values and mental wellbeing. The chapter is therefore organised into four sections, respectively. These themes provide a conceptual framework for underlying respective sub-themes. The chapter concludes with a closing statement which briefly summarises the key findings.

\subsection{The "average kiwi man"}

There were three themes that stood out from the participant descriptions of an "average kiwi man": generational difference, Richie McCaw and the All Blacks, and the student. These themes are discussed in detail in what follows.

\subsubsection{Generational Difference}

Chapter Two reviewed how the "kiwi bloke" has been a dominant masculine archetype in New Zealand for some time (Sim \& Thomson, 2000; Joseph \& Falcous, 2017). It is a model that was established through a complex interplay of historic events and geographical/environmental circumstances. Over time characteristics of the "kiwi bloke", whilst consistently identifiable, may have changed and evolved. Six participants in this study discussed a "generational" element to the "kiwi bloke" and described it as a stereotype. The use of the word "stereotype" highlights the participants acknowledgement of the "kiwi bloke" being an oversimplified representation of the average kiwi man. However, sometimes participants wavered between the use of the word "stereotypical" and "typical". The generational difference was discussed by participants in two contrasting ways; the "lifecourse" and "changing times and expectations".

\section{The life-course}

The concept of the life-course entails that at different stages there are certain expectations surrounding what a man should do. It illustrates how certain practices associated with the "kiwi man" are represented at different times in a man's life. One participant, Tom, attributes his description of the "kiwi man" to an older man: 
I think there's definitely the, um, I suppose, in an older, older man, you've got typical like, um, "follow the rugby" um, drinks beer, kind of typical like stereotypes.

Tom's discussion indicates that he was referring to something that he perceived to still exist, but he was yet to experience. I found that participants referred to different stages in their lives, beginning with their younger years and college:

Especially when you're growing up as a boy, there's a lot of pressure from society to stay in things like sports.

I think sport, representing in sports, is something that's carried through heaps like in college that was like a big thing...we were quite an artsie college but it was still like if you didn’t play rugby you didn’t really get the light of day that much.

Definitely in high school there was like a big push...you'd play football, you'd play rugby....and it's just what you did...I'd say once you get to university everyone just sort of chills out.

These participants highlight that participating in sports in their younger years was what was expected. They describe things such as a "push" or "pressure". The second quotation provides an important contrast because it shows that even in an "artsie" college, this participant still felt that sport was a priority. Connell (2000) has noted that schools can be a primary site for symbolic representations of masculinity to be formed against a wider cultural "backdrop". Considering the importance of sport in relation to hegemonic masculinity in New Zealand, it was interesting to hear about the participants' description of the prevalence of sport at school. It seems, however, that this pressure dissolved as the participants left school and moved into higher education.

Subsequently, drinking was discussed as something which becomes a general expectation in the late teen and university years:

As you go up through high school everyone starts drinking at what like year 11 year 12, and then, you know if you're not in with that crowd it's a little bit, you know, socially isolating.

There seems to be a bit of a party culture in the sense that yea, it's expected that once you reach an age you should be drinking. 
Tony and Harry discuss how at a certain age drinking became a normalised practice. Willot and Lyons (2012) discuss how beer or drinking is often a symbol of an individual's entry into manhood both in New Zealand and other western societies. Different contexts have established rituals or practices whereby a young person will experience their first alcoholic drink. For instance, it may be a tradition for a father to share his first drink with his son. However, Tony's description shows a less symbolic or traditional version of expectation which stems from peers. Tony perceives this to be a normalised behaviour for that age group, and if it is not mirrored by an individual then it can become socially isolating.

Drinking behaviour was for different age demographics was discussed by the following participant:

I suppose...the younger orientation will still have the same like watch sports, um and drink, but also have a different, like they won't just watch sport...they might end up just getting drunk or just partying and still living the young life kind of thing.

The participant describes the differences in drinking behaviour between and older and younger men. He discusses how the younger orientation may end up "getting drunk" and "partying" compared to simply watching sport and having drink which he associates with an older group. This "younger orientation" is something that was often referred to by participants as "the lads and the boys":

You've got the typical kind of older generation, but I reckon the younger's very much kind of the "lads and the boys" kind of orientation...there is a bit of a change.

This terminology is descriptive of a particular "phase" or association to a group of men. The term "lads" originated in England in the mid-1990s and has been described as a dominant form of masculinity that involves "having a laugh", playing sport, wearing the right sorts of clothes and a rejection of academic hard work (Jackson, 2010).

\section{Changing times and expectations}

The idea of changing times and expectations contrasts with the previous notion of progression throughout the life span. The concept suggests that the idea of a "typical kiwi bloke" and all its associations are a thing of the past: 
uhm sporting I suppose I don't know that's hard it's it's kind of changed now your average kiwi man is probably the person you think of back in the 1980s or something it's probably a bit old school for me.

This is interesting because Tristan identifies that what he thinks of as "an average kiwi man" is not something that, from his perspective, would fit a current description of most men in New Zealand. However, both Tristan and other participants did not provide a current or alternative description. The failure to provide a current description could relate to Gee and Jackson's (2012) discussion on how as "traditional" values and traits associated with masculinity become less applicable, new conceptualisation go through a struggle towards becoming concrete. The following participant describes how traditional expectations are still there, but from his perspective they are lessening:

Um, nowadays it's less, there still is a bit of an expectation to be honest to, you know, go play rugby or, you know, go to the farm, have some fun on the farm, but it's a lot less than what it was a couple of years ago, 20 even, 20 years ago....The, you know, just "harden up" and all those sorts of things, that's still a bit of an expectation. Especially if you've got like older parents, who were raised in that sort of generation.

This quote provides insight into the passing down of certain qualities from generation to generation. Julian discusses specific practices that may have been more prevalent twenty years ago, but he also highlights that "harden up" is still something that is expected. Here he is referring to something that is less of a specific practice and is more of an underlying social dialogue. Preferences for specific practices such as farming or rugby are perhaps more susceptible to changing times and expectations, but underlying rhetoric's that are built within a culture are harder to clearly observe and therefore change.

\subsubsection{Richie McCaw and the All Blacks}

Richie McCaw is an ex-captain of the well-known New Zealand rugby team the, All Blacks. Interestingly nine participants in this study stated that McCaw was someone who they viewed as either an embodiment of the "average kiwi man" or a prominent role model for men in New Zealand: 
Aww Richie McCaw probably. I reckon cause like, um it's just like what you'd call a top bloke. Reliable, honest, um leading the country in a positive way in something we are good at...Yeah, I think people look up to that for sure.

Here, Daniel discusses why he thinks Richie McCaw is a role model for men in New Zealand. He describes various qualities including the fact that Richie McCaw has led the country. There was an interesting juxtaposition between participants discussions of an "average kiwi man" and their discussion of All Blacks players, because leading the country as an All Black or Richie McCaw would be unattainable for the average man. However, there was not always a clear separation between discussing the "average", or "typical", kiwi man and discussing All Black players:

Well, not necessarily like a specific person, but like I guess like an arch type would be, um like a rugby player... I think people's conception of a rugby player fits that, you know, that stereotypical Kiwi bloke.

This quote further highlights the position of a rugby player as being a representation of the stereotypical kiwi bloke from the participants' perspective. A strong tie was made between rugby players and an average kiwi man or bloke, and it seemed to be the only obvious "marker" participants could provide as a symbol of representation. The following participants emphasize the influence this has on how boys and men position themselves in New Zealand:

The role models that we show boys...sports people or people who are very physically active and um, I think that makes a big impact on young kids...um, so it's, it's like one of those social markers like, um, like sport that like becomes part of the ideal male figure.

Um, I suppose you could go off all the stereotypical stuff like be a tough guy, be you know, strong...I mean essentially just be like an All Black, like tall, handsome, muscular, all that. That would be like your ideal. Even for the average I feel like that would be the standard most people would hold themselves to, you know, like look at what's around and then try and achieve that.

The concept of sportsmen being the "ideal" was emphasised by many participants, which is illustrated in the following value judgements:

I would say that Kiwi men look up to sports people more than politicians. I think it's a very easy opinion. 
Like if you're looking up to people, you're not going to be looking up to tradies, you'd be looking up to you know sports teams and even like the black caps and stuff like that.

Here, Tony and Daniel discuss sports men or teams in relation to other roles in society; the "tradie" and a politician. It illustrates the value of sportsmen over the value of other role models or positions in New Zealand society.

Three participants discussed other 'embodiments' or 'role models', however, they were also discussed as idealised examples. Tom, made the comparison between a "kiwi dad" and the All Blacks:

They've kind of been like role models of Kiwi culture.... Where, um, the average kiwi, you know, of course, most Kiwis aren't famous. So, it's different. The way I'm envisioning it anyway is that they're two kind of, you, you look up to them in different ways...But yeah, the average Kiwi dad...I'm thinking of like this really cool, genuine fella and his kids, um, in his forties and he's just um, happy and open to chat.

Tom provides an interesting comparison between two types of male role models. The way in which he describes the "Kiwi dad" from his perspective is a more average or possibly attainable version of a role model in comparison to the All Blacks. However, he is still describing the "average kiwi dad" in a distinctly positive way. Two other participants discussed alternative role models, the first being Harry who discussed a friend of his who was head boy:

Uhm, he was our head boy. So obviously that uhm, embellishes a lot of things cause he got made head boy.

Something that tied Harry's description of his friend, Tom's description of a "Kiwi dad" and the various descriptions of the All Blacks is the idea of success and the attainment of something that others would want. The last participant that stood out in this category was William:

I mean when you say an average Kiwi man for some reason, I'm always thinking of like an Anzac Day soldier or something weird like that (laughs)...Even though it's nothing of the sort. 
This stood out because of the simultaneous similarities and stark differences between the role of an Anzac Day Soldier and the other role models provided. It should also be noted that the interview for this participant was conducted over a month prior to Anzac Day in New Zealand, and therefore it is unlikely that this factor would have influenced his answer. An All Black and an Anzac Soldier can be described as a "hero". Both roles require great displays of strength and the representation of their nation. However Anzac Soldiers are often not known or recognised, and their role requires a great deal of sacrifice. Whilst becoming an All Black player, regardless of identity, would make a man a hero in the context of New Zealand culture, they differ from soldiers in that they are long-standing idealizations of what a New Zealand man ought to be.

\subsubsection{The Student}

Having found that sports heroes were the dominant idealisation of what it is to be a "kiwi man" in New Zealand, it was interesting to see how being a student would align with that idealisation. As discussed by Banner (2005), historically the "kiwi bloke" raised questions about the masculinity of intellectuals. In sections 4.2.1 and 4.2.2 the emphasis participants place on physicality and drinking is discussed. Interestingly, those practices contrast with academic practices which most participants felt were not masculine. The following two participants discussed reading:

I know, I just don't seem like the type that reads, and that's the, that's, the biggest issue is I don't fit into the lads, but I don't fit into the academics, cuz I enjoy the academic side but I also enjoy getting drunk and having fun.

I loved to read, you know, I did feel bit alienated from the model because I preferred to read Philosophers Stone rather than like playing cricket.

In both instances Tristan and Mike refer to reading as a practice that is associated with a type of person or role. In the first instance, Tristan insinuates that reading is something associated with academics and it is something that he enjoys doing. However, he does not feel as though he is the type that reads and enjoys getting drunk which he therefore does not associate with a practice of an academic. Although Mike is not directly associating reading with the role of an academic, he is discussing how reading separated him from the dominant model, simultaneously insinuating that this model revolves around playing a sport or in this case cricket. The following participant, Harry, referred to the idea of a "main social group" which aligns with a dominant model: 
If you were a nerd or geek, if you studied or, you know, you probably weren't in the main social group.

Here, Harry is talking about his time in high school, which Mike was also referring to in his discussion above. However, there were participants who specifically discussed being an academic or intellect in the present tense:

I don't think that uhm, academic things make you feel masculine or manly...I think you miss, I think the sense of community is incredibly important in terms of feeling manly or feeling masculine like, um, being accepted by men and being relied upon by men and just doing all this like, um, insular work that isn't necessarily conducive to that.

Daniel brings up an important idea here, he is discussing how the insular work that takes place in academia does not foster a community that reinforces masculinity. This ties into the importance of social bonding (4.4.1) and the idea of loyalty (4.3.2). But it also links back to Harry and Mikes discussion above. Both participants indicated that reading and studying in high school was something that did not include you in the dominant model or main social group. It was also found in section 4.1.1 that participants felt there was a significant pressure to play sport in high school. This then presents a potential tension or "turn around" in expected practices when men transition from high school to higher education.

The literature review highlighted that although attending university is now commonplace in contemporary society, there is a possibility that there is still pressure for young male academics to try and emulate dominant masculinity in some way. The following quote is an illustration:

...they definitely have to do those types of male activities cuz this sort of uhm social conditioning is so strong that you can't really just be a smart guy and like that's enough in your man hood, like you'll be seen as a nerd and so you've gotta sort of buff it up in some other way.

It's, you know, being an intellect isn't as masculine. Um, and if you are trying to be masculine within the university and then I suppose you find friends or like the people around you, you find um, friends and hang out with people that still try to align with that.

These participants discuss either having to "buff it up" or find friends who "align" with more masculine ideals. The other "male activities" Craig refers to here are those surrounding 
physical activity and drinking. He discusses how being a smart guy would not be enough to solidify "man hood" and would result in you being labelled a nerd. This insinuates that the label of a nerd could result in someone being viewed as "less of a man". Tom similarly describes being an intellect as less masculine, therefore if you are trying to be more so you will find friends who align themselves with masculine ideals.

\subsection{Cultural Practices}

The concept of the "kiwi bloke" is mostly centred around sport and drinking (Joseph \& Falcous, 2017; Willot \& Lyons, 2012). Interestingly, these practices were very prevalent across all the interviews in some way. The practices were sometimes discussed in combination, as illustrated by the following participants' response to the practices of an "average kiwi man":

Stuff like going into the outdoors, camping, hiking, um, you know, having a couple of beers all that sort of stuff. Yeah. I don't really know what else, what other activities.

Play rugby, uhm drink some beers, uhm maybe go on a tramp every couple of weekends, go fishing, you know outdoorsy.

These quotes highlight the combination of drinking and physical activity as the most recognizable practices participants associated with the "average kiwi man". One participant, Joseph, made the connection between the All Blacks and drinking:

It was known that like the All Blacks they would finish their games and then go on to the drinks... and then so that probably has like a flow on effect at the grassroots game would be more inclined to having drinks after the game and stuff like that.

This further illustrates the tie between sport and drinking. It is also another illustration of the participants' perspective on the influence of the All Blacks.

Both physicality and drinking were subsequently highlighted in varying ways by participants which will be discussed in the following subsections.

\subsubsection{Physicality}

The participants all discussed physicality in different forms in relation to the "average kiwi man" and in combination with drinking. Historically, the "kiwi bloke" is associated with the physicality of the pioneering lifestyle, the conquering of land and the importance of physical 
labour (Sim \& Thomson, 2000). The following participant discussed a historic parallel between men and women:

I think socially that might be where there's a bit of a parallel between men and women in New Zealand. I think maybe women stereotypically would have more academic jobs or academic lives and men would have more physical... New Zealand is quite progressive in that sense. That might not be completely accurate. I think historically there might've been more of that sort of social parallel.

Here, Seth discusses the social parallel between men and women, highlighting that physical jobs have been historically placed on men. Jobs such as farming and being a tradesman were also discussed by participants, alongside physicality generally:

I guess if I'm trying to think of the average man in New Zealand, probably, you think trades, you think uhm...DIY, building, cars. Uhm I don't know, yea I guess sports. More hands-on activities is what I would think.

Like the, the sport and the farming and you know, all the hands-on stuff is definitely surrounding the sort of concept that men get their hands-on things and do things.

Both participants discuss the physicality they associate with the "average kiwi man". Participants discussed physicality as something that was not just associated with their perception of the average kiwi man, but they also highlighted a more general societal expectation:

Social conditioning and culture is a big part of socializing men in New Zealand...like a man should be strong and domineering and heroic and adventurous and "out there doing things".

Um hunting, fishing a lot more of like the, the quote on quote "manly things", you know...like going into the outdoors, camping, hiking.

These quotes illustrate that the participants see physicality as part of what a man "should" be doing. The first quote interestingly ties into the role of the media reinforcing a dominant image of masculinity. Media influence is also something that was mentioned by a participant in relation to beer advertising, which will be discussed in section 4.2.2.

One of the main physical activities that was discussed by participants was sport. I previously highlighted that the expectation to play sport was something participants discussed as 
prevalent in their high school years. Two participants also highlighted that sport was part of being accepted:

In terms of a teenage kiwi... obviously from what I know it was very much a, you had to play sports to be popular. It was pretty much the only thing that was like, um, accepted to do.

Yea I think it's kinda hard if you don't do a sport of some sort about fitting in... we were like the music people, group, at college but I also play cricket. So I've sort of found a way into those other male groups from playing, doing sport and being able to talk about that sort of thing, whereas like the other guys found it very hard.

Daniel and Harry both discuss how playing sport in high school was something that enabled men to be accepted into social circles, with Harry specifically identifying that it was something you had to do to be popular. Daniel discusses how by playing a sport he was able to "find a way" into that group, which is contrasted against his music friends who did not play a sport and as a result were not accepted. Sport therefore acted as a type of "social glue" defined the boundaries between different social groups or even a social hierarchy. Another participant also discussed this concept but in relation to following sport as a New Zealander in general:

There is a bit of an orientation of like "do you follow the rugby?" ...Um it goes all throughout I suppose, um, New Zealand as a genuine talking point....it is a way of like making, um, making new friends...But yeah, the downside comes when you're not interested in following sport.

Again, Tom is referring to sport as a social glue, something that enables men in New Zealand to bond and connect. He specifically referred to rugby, and throughout this particular area of discussion he also discussed "following the All Blacks". Rugby has historically been a very significant part of practicing dominant masculinity in New Zealand and New Zealand culture in general (Joseph \& Falcous, 2017). This was recognised by participants:

Um, I mean realistically with New Zealand loving rugby, I think rugby is a big thing in New Zealand. I think it's one of the similarities. It's um, yeah, I think there is a big expectation in terms of masculinity for men to watch rugby, you know, across, you know, being a lawyer, being a farmer, being whatever. 
When we think of New Zealand people think of like rugby so that's engrained in our culture and then that's what sort of sets us apart from the world. So, it's sort of expected that you play a sport, that you play a role in actually being Kiwi and actually being a part of New Zealand culture.

Julian and Joseph both highlight the importance of rugby to New Zealand culture, but they also establish a connection between Rugby in New Zealand and the expectation placed on men because of this. However, there is a slight difference in the insight that is provided in these quotes. Julian discusses the importance of watching rugby which is different from physically participating in the sport. Whereas Julian discusses the expectation of playing sport. Both participants highlight that rugby is something that is inherently part of being Kiwi and therefore the expectation to be involved with the sport in some way goes across individual identities.

\subsubsection{Drinking}

Participants associated drinking with the "average kiwi man". It was also a practice that all participants discussed. In the context of the "kiwi man", participants specifically referred to drinking beer. One participant, Julian, made the connection between the "Speights" beer advertising campaign and the social construct of masculinity:

You get advertising for like Speights... and it might have, you know, a man there drinking and it's that social construct again, it's just, it just appears out of nowhere really. It's just there and it's like very weird.

Here, Julian was discussing where the expectation to drink in New Zealand comes from. I found the way Julian describes this relationship interesting because it highlights his awareness of the subliminal connection between beer advertisements and the "kiwi bloke". The literature review section discussed how beer brands in New Zealand symbolise the dominant ideals of a "kiwi bloke" in their advertising (Penwarden, 2010). Another participant highlighted that beer is the drink which is associated with the start of a binge drinking culture in New Zealand:

I mean that was the whole, was the whole law around, uh, with, whether pubs stopped supplying beer after six or which is, which is kind of where people seem to think the whole binge drinking culture started...there's a lot of, at least beer related alcoholism in New Zealand, probably a lot with men. 
Alongside discussing beer as the historic beginning of "binge-drinking" culture, Seth also highlights the current beer "binge-drinking" culture in New Zealand. He initially acknowledges that women are also a part of that culture but relates beer-drinking alcoholism predominantly with men. The "binge-drinking" culture in New Zealand was discussed by five other participants in predominantly negative ways:

New Zealand has a very toxic drinking behaviour, a lot of us are binge drinkers...Like go to the store, buy a, you know, box and you finish it within the night... and we've got stuff like crate day, you know, which it's really bad for us.

I mean overall, considering our culture is a binge drinking one, it's more drinking to excess, which is by definition too much. Um, which has overall negative side effects and I would say is a detriment.

Both participants discuss how they believe the drinking culture in New Zealand was negative. I found that when discussing mental wellbeing most participants did not consider drinking to be a conducive to positive mental wellbeing. However, it was discussed as a potential outlet for emotional intimacy or an emotional vent.

Participants also discussed drinking as an entry into socialization:

If you don't include yourself in binge drinking, um, that can be a social, um, hindrance and that really impacts you because we're social creatures. You want to be, um in a social group.

Therefore, the participants perceive there to be a tension between the negative effects of binge drinking that participants discussed and the necessity of binge drinking to form social bonds. Participants also discussed drinking and bonding as being specifically related to their university experience:

Oh definitely yeah you can't really get through university without sort of, it's just the way you meet so many different people, it's just binge drinking culture, and you know it's almost as if, if you don't sort of binge drink at the weekend you don't meet a lot of people and stuff.

I'm just thinking back to first year, it was like, probably the only thing that made me feel welcome with my male friends. 
There is an interesting parallel between participants' discussion of sports as an entry into social groups in high school and drinking then playing a similar role in university. Drinking was the only practice that was discussed by participants in relation to university and masculinity. As well as being discussed as a "social glue", it was also discussed in terms of an expected practice at university:

Yea there's certainly like a pressure, not just like a masculine pressure but there's sort of like a university pressure to do that type of activity.

I was talking to one of my cousins who finished uni a couple of years ago. It was like "You didn't go out!? You didn’t drink?!" and you know, "tell us all your wild stories" and that kind of stuff.

It is interesting that these participants felt there was a distinct pressure to drink as part of the university experience since it contrasts against the lack of pressure for young men to be focused on academic activities discussed in section 4.1.3. It was apparent from the various discussions provided by participants surrounding drinking that this practice presented multiple levels of tension.

\subsection{Values}

Four themes were identified from participants' discussion on values. These four themes are discussed across two sections in pairs, due to relatedness but also a simultaneous contrast. The pairs of values are; future family values vs values of emerging adulthood, and loyalty between men vs self-reliance.

\subsubsection{Future family values vs values of emerging adulthood}

Five participants identified family as something that people would think was important to the "average kiwi man":

It's probably the concept after uni or whatever you're gonna do you have to have a partner and you have to have a family.

Here Tristan refers to having a family or partner as something that a man is supposed to do when he is older or has left university. This aligns itself with the life cycle concept discussed in section 4.2.1. The other participants who discussed family also discussed it being something that you work towards as you get older. Two participants referred to working towards the "kiwi dream" as something which was valued: 
Oh, I think it's in terms of like we, they or us, where we want to be at the end of our lives or after a career is to have like your own little section and your own sort of do it yourself little paradise. I think it's the Kiwi dream. Right? ...it's like the American dream and the Kiwi dream are kind of like similar in a lot of ways.

I suppose family life is also a big part for particularly older kiwi males. And uhm like moving towards like the kiwi dream...having like a house and kids and a wife... it might not just be an influence of kiwi culture, but could be an influence of kind of just, you know, "house and family and kids" and kind of the movie in America.

These descriptions are important as they provide a description of a family structure, with both participants referring to America. This concept surrounds the idea of a "traditional family" or "nuclear family" with the father often positioned as the breadwinner. Four out of the five participants specifically discussed being able to provide for a family:

Being able to provide for a family, um, being able to sort of like maintain a house, maintain a car, maintain a job.

The above statement was from a participant named Seth, who subsequently acknowledged that this was a historic expectation placed on men but in theory it is placed on both sexes in modern day.

Historically I think that's been laid on men more than women, I think modern day maybe not. It's at least in theory shared more equally between both.

Interestingly, this statement also relates to the concept of generation discussed in section 4.1.1. However instead of the life course idea, Seth's discussion surrounds the concept of providing for a family as a historical factor expected of men that is perhaps changing.

The contrasting concept of "values in emerging adulthood" was only explicitly discussed by one participant. However implicitly most participants in their discussion provided a contrast between career driven and family goals with drinking and "laddish" behaviour. One participant, Harry, discussed the idea of short-term values which align with his age group:

Deep down there's probably some core values of whānau and family...although when it comes up from that it can get murky because as you're younger you tend to do a lot of things that make you feel happy...I value things like my education and longterm plans...I can very clearly see that that's a bit of a divide between me and my friends....(who) very highly value, um, yeah, having fun. 
Harry provides an interesting contrast to the previous concept of the "future" value of family. His description alludes to values being something that grow over time, with the younger years being orientated around the value of short-term gain. This aligns with literature that examined value change across the life span and showed that sensation seeking, and novelty are more typical of young people subsequently decreasing with age (Gouveia et al., 2015).

This idea was also implicitly alluded to by other participants, particularly when it came to the change from the beginning of the university to the end. The following participants illustrates a discussion of this change:

I think when you go into a place like university, um, where maybe the average male is more into "lad" things...there's probably a lot of students whether they agree or disagree with it that would also follow that lifestyle just because that's the sort of majority that's the trend...I mean my friends would be finishing their degrees...that's when you sort of, I would say you start to form your own values.

When Seth refers to "lad" things he is referring back to his previous description of "drinking, town, parties, girls". Whilst he does not state the type of values that form towards the end of university, he insinuates that values associated with "lad" things diminishes. He also highlights another important idea surrounding the "majority". He attributes the value of "lad" things to a trend or majority at a specific point in the early stages of university. This idea of "majority" value has synergies with wanting to feel valued which was touched upon by Harry:

I think one thing every teenager feels at some point is wanting to be valued...If someone wants to hang out with you, that kind of stuff seems to be very important.

This is an interesting concept because it moves away from attributing value to a practice and wanting to feel important and valued intrinsically. Harry highlights that "someone wanting to hang out with you" is important which in some ways presents an idea of a "hierarchy of values". Valuing things that the majority may explicitly value at a specific time may serve to reinforce an implicit value surrounding inclusion.

\subsubsection{Loyalty between men vs Self reliance}

Five participants discussed loyalty between men as something which people would perceive to be a value of "the average kiwi man". However, it was discussed by participants as something that they also valued and experienced. Interestingly this was mostly tied into a physical sense of loyalty: 
It's just nice to know everyone's got your back in a physical sense, I guess.

Even when participants did not explicitly state that they were discussing loyalty in the physical sense, their discussion still centred around an activity or doing something:

If there's something I need done, I can rely on this other dude to help me out...I think that's ah, different kind of currency of like, I do a job for you, you do a job for me.

Just uhm like participation, really big around if all the boy's do something you've gotta do it as well.

The focus on physical loyalty here presents a potential juxtaposition between loyalty and teamwork, and self-reliance or "do it yourself". The following participants discussion of values positions these concepts side by side:

I think being strong and stoic and sucking it up and carrying on by themselves uhm, self-reliant, self-resilient uh loyalty always looking after your mates.

It should be acknowledged that these values are not mutually exclusive. However, the discussion around loyalty and teamwork was only discussed in relation to physicality. There are two different ways that self-reliance was discussed. The first was the concept of "do it yourself" which surrounds traditional expectations of being physically capable:

They say that number 8 Kiwi wire... actually being able to solve problems on your own and not having to resort to going to other people and asking.

Fix it yourself and that's seen as a respectful thing...like this is your job you shouldn't make it someone else's.

Both participants are referring to being individually competent when it comes to singular physical jobs and issues. The first participant, Joseph specifically refers to the "number 8 kiwi wire" which is representative of a New Zealand mentality surrounding the ability to repair or create objects using basic material. The second use of self-reliance was discussed by all participants in this study and revolved around the expectation for men to be mentally self-reliant:

I suppose you do have a bit of a bias towards expecting guys to be able to handle it a bit more or not, you know "whine" a huge amount or anything like that. 
I feel like men don't want to offload their problems to one another in terms of like having a bad day, you know it'll just be like "toughen up" or you know "you'll be right".

Interestingly, these discussions present themselves very similarly to the previous statements regarding physical capability. There is a sense of "not offloading your problems" and being able to handle things on your own. However, these participants are referring to the ability to deal with internal problems independently. This ties into the discussion surrounding emotion in section 4.4.4. The following participant was the only one to describe relying on friends for emotional vulnerability:

All that stuff about teamwork and things that only applies to like "the boys" or the men who have like made it into that model...the, the male friends that I have, yeah are very different from that model... as a whole my friend group we value like, I guess like emotional honesty, um, vulnerability and I guess intimacy.

Mike describes teamwork as something that only applies to men who have made it "into that model", the way in which he described this model had synergies with the physical sense of comradery that was described by the other participants when discussing physical loyalty.

\subsection{Mental Wellbeing}

Five themes on mental wellbeing were identified; social bonding vs social ostracism, banter, competition, undermining emotions and drinking as an outlet for emotions and intimacy. Encompassed within each theme is the discussion of their function to potentially enable and constrain mental wellbeing. Each theme also addresses the relevance to the context of higher education.

\subsubsection{Social Bonding vs Social Ostracism}

There is a scarcity of research which looks at perceptions of masculinity and its facilitation of mental wellbeing. One project highlighted earlier was an Australian project "Men's Sheds", which brings men together to work on physical projects to alleviate feelings of loneliness, (Woosler, 2018). Eleven participants in this study identified that the social aspect of the practices they had discussed were positive for mental wellbeing. Most participants in this study identified the positive effects of sport or physical activity in creating social bonds:

You bond over, like, how bad the environment is, like if it's pouring down with rain and you're tramping or playing rugby and you're just like "this is pretty $\mathrm{sh}^{* *}$ " and that's like something you've got in common all of a sudden. 
Connection is huge aye, as soon as you're doing a sport based or team-based exercise and you do make connections that way...I felt connected because I was able to relate to people on that level.

These participants both discuss the benefits of participating in team-based physical activity. The first participant is specifically addressing bonding through adversity and physical challenges. Therefore, from his perspective, it is not necessarily the specific sport, but the context of bonding together in the face of physical feats. Whereas the second participant is highlighting the element of sport and exercise that provides an area of common-ground and gives him the ability to relate to other men. This idea of relatability was discussed by another participant:

I suppose like uhm fitting in with the group there's something to bond over. So like most Kiwis follow rugby kind of thing. So, if you're also following rugby you can easily like go into a bar and make a new friend or just bond with another Kiwi male over sport in particular.

This participants' discussion differs slightly from those highlighted above. Instead of physically participating in sport, he describes the fact that simply following sport is something that can help men bond. Participants also related drinking to the formation of social bonds. The following participant discussed the function of drinking as part of socialization at university:

I guess the problem is with drinking is it's just mainly it could be especially for uni students it's like the main social interaction for the week so it's your chance to actually talk to one another especially in a more relaxed environment.

This participant highlights that drinking is problematic but simultaneously facilitates a relaxed environment necessary for socialization at university. This description aligns with most of the participants' views on drinking and socialising. They acknowledged that drinking was problematic because it had the potential to cause harm or be relied upon in an unhealthy manner. The functions behind alcohol as a social bonding facilitator is an emergent area that is being explored by alcohol researchers and social psychologists (Sayette et al., 2012). There is a potential tension between the need to drink as a social lubricant and alcohol becoming relied upon for social and emotional intimacy.

Participants in this study identified the negative potential that drinking can have on mental wellbeing as something that outweighed its use for social bonding. 
Two participants discussed how the university facilitated finding their place outside of these dominant practices:

I think being a student you also find cliques and when you find your clique at university, that's your positive "finally I'm in" and I defiantly had moments of that at Uni. I think a lot of people do, especially men who are just a bit tired of the average.

I think it depends...like what groups you find yourself in...the arts and in psychology it's generally quite progressive and open. So I think that's had a probably a positive impact really being a student and being, and being a man for me, just in terms of what I've studied, um, because what I study is generally supporting the idea of like being vulnerable and you know, positive mental wellbeing.

Whilst some participants perceived that being a student may not necessarily align with the dominant practices of being a man, these participants highlight how university may also facilitate finding a social group with shared interests outside of the average practices of men at the national level.

The importance of finding a social group or establishing a social bond was emphasised by the following participant:

I think as a young man you want to be welcomed into a wider group of men, right? Like whether that's in a sports team or like a group of friends... And if you don't get that community, then like that's, that's where the mental wellbeing falls apart, is not being able to find strength and identity with other men and you start resenting being a man or you start like, um, the words not knowing your place comes up for me. Yeah. Yeah. And that's when you're like, I'm weird, I'm this and that. And it's like, um, fear and shame is the big underlying, uh, feelings, I would say.

Daniel makes a distinction here between the importance of finding your social group and the negative effects of not being welcome or finding your social group. He also specifically discusses the implications of feeling shame and fear. Not being welcome entails that there are certain criteria that an individual must fulfil to be allowed into specific groups. All of the participants in this study acknowledged that there were expectations associated with being a man in New Zealand or being a man generally. The most distinct expectations have already 
been highlighted; the expectation to play sport in high school and the expectation to drink at university. However, some participants specifically discussed the negative implications associated with having that pressure and expectation:

I think most men feel the pressure to act in a certain way that aligns with that stereotype uhm definitely in New Zealand...the pressure is enormous!

From what I can understand, that's the whole idea of toxic masculinity is the fact that there's sort of a societal expectation for men to be a certain way or to be able to do a certain thing. And I guess that's not great for your mental wellbeing if you're not able to fulfil those expectations.

I think overall there is a lot of pressure on guys to do a lot of things... so you've got to consider it, you know, what do you actually want to do versus what are you told to do...if you aren't prepared for that and you aren't aware of how to properly analyse all of this, it can definitely have a negative impact on your wellbeing.

Each of these participants are discussing the pressure and expectation for men to act in a certain way. Their discussions all slightly differ, however, they all emphasized the negative potential of this expectation on mental wellbeing. The second participant described "not being able to fulfil those expectations" as something that would negatively affect mental wellbeing. Most participants in this study referred to either an "in-group and out-group" dynamic or the idea of being "pushed out". This is inextricably tied with the presence of expectations in society because if one fails to meet those expectations there is a risk of being socially ostracized. An example of the expectation to binge drink was detailed by Harry:

If they're having their parties and you're the one telling them "maybe you should drink some water." They don't drink the water and then you don't come to the next one. So yeah.

Here Harry provides a clear example of being ostracized by going against the common practice of binge drinking. He describes how telling other men to drink water, would result in being excluded from the next social event. In discussing this scenario, Harry is essentially separating himself by referring to other men as "they' re" collectively: "if they're having their parties" as opposed to "when we have parties". Similar terminology was used by another participant, Mike: 
...they're working within that model, obviously a lot, you know, feel alienated from that model and, and you know, those are the people that are very heavily affected by it.

Here he says, "they're working within that model". The following participant used similar terminology but in the opposite direction:

cuz some take pride I spose, especially now there's a whole lot of "you can be who you want" so people take pride in "well I wanna be me" whereas before it hasn't really been an option, and that's cool for them but I think sometimes taking that option ostracizes yourself from, too far from every day society.

This participant perceives that going against the majority is something that can ostracize you. He discusses the idea in a way which insinuates he disagrees with the risk associated with being different or individual. Therefore, there is a possible tension when it comes to addressing mental wellbeing or showing vulnerability:

I feel like men have no other choice, its either you go with all other men and male solidarity and you drinking games and your rugby matches or you don't have friends and you're kind of ostracized. And so I feel like men are stuck between a rock and a hard place they're either very emotional and they go against the entire grain of society... or they conform and they kind of lose a part of themselves so I think it certainly helps them having friends and mates and stuff uhm in a way that people should and that probably helps as a human but I think they also lose part of themselves along the way.

The findings that surround emotions, specifically, will be discussed in section 4.4.4. However, Craig's statement is important here, because it illustrates the polarisation of the practices that constrain showing emotions and drive social ostracism. He highlights that both "sides" have positive and negative implications.

Earlier the literature highlighted that individuals who were "confident or self-assured" were able to simultaneously endorse and challenge dominant masculinity (Harris, 2010; Hong, 2000). Interestingly, two participants in this study discussed this concept;

The luckiness for me is that I've never felt the need to join a group or all that, I go where I want to and I enjoy it....I'm confident, and I've been good at sport, I've always made friends I can speak to people you know I'm lucky that I've got the skills that I can just talk to anyone. I don't care, and I'm confident so yea I'm 
just lucky that I've grown up that way. But some people, I can understand that some people don't have the courage, or they just don't feel comfortable."

I think it's about, um, confidence. I think people, as soon as you see someone that's confident and seems like they know who they are and talking to you can't help but admire and want to be around this person.

Both Tristan and Daniel were participants that acknowledged the separation between different groups of men and they both acknowledged that potential negative impact of certain societal expectations. However, both expressed themselves as people who can move between groups which they both subsequently stated was due to their confidence.

\subsubsection{Banter}

Six participants referred to what they called "banter" or "rip culture", described in detail by the following participant:

Like um, well I mean, it can take a few different forms, but like, like kind of jokey insults, you know where like, if someone wrote it down, you might think "aw that, that's like bullying going on." But within that context, everyone involved might be like having a bit of a chuckle about it, although of course, sometimes feelings can get hurt in a way.

Here Mike describes "banter" as something that would be humorous for those involved but indicated that sometimes feelings can get hurt. This description aligns with previous research that discusses the function of banter providing both a bonding capacity but also an aggressive and competitive capacity (Hein \& O’Donohoe, 2014; Dynel, 2008). The following participant focuses on "rip culture":

Um, yea for guys, um, it tends to be, tends to be a very common Kiwi male thing, is within your friends group, you rip each other, you, um, you know, every week you pick some guy whose going to be the butt of everyone's jokes for the week. $\mathrm{Um}$, and some guys get it more often than others within a friends group.

This participants' description highlights a specific dynamic in how the jokes are target rather than always simply a group "back and forth" behaviour. His description leans more towards "picking" or "targeting" than a behaviour that facilitates social bonding. Daniel discusses the risk of "rip culture" and how it could have something to do with establishing "us and them" boundaries: 
What's big for me about this whole topic is this idea of what I call like rip culture and with how Kiwi men interact with each other is to like rip on each other as a joke. And I think that it's really easy for those jokes to turn sour. Like uhm talking behind your back about, "oh this, oh $\mathrm{f}^{* * *}$ this dudes such a fucking little $\mathrm{s} * * * . "$ Or "he's such a little fairy" or like, or whatever. Like I think that there's this, yeah, this, I don't know what it is, maybe it's just like, an "us and them" sort of like... you know, like, um, cause it's sort of like I'm teasing you to get to, to make you fight back and that's how I get your respect.

Daniels emphasises the capability of these jokes to be hurtful. Interestingly, his description also includes elements that tie "rip culture" into other categories. For instance, he discusses "us and them" which relates to the discussion in section 4.4.1 and the dynamics of social bonding and ostracism. He also discusses how the teasing can be used to make another person fight back and gain respect. This relates to competition, the theme to which we turn.

\subsubsection{Competition}

Ten participants in this research discussed competition. This was not raised in relation to the "average kiwi man", but something that was related to masculinity generally:

I spose thinking of the wider concept of masculinity it's a competition "I wanna be better than the next guy so I've gotta out do them" this stupid thing that happens.

Oh, I think competition's big aye between each other and between all the other dude's like we have to be like, yeah, we've gotta be like, aw what is it, we've gotta to be better, I've got to be better than them, I've got to be stronger and like, yeah, rark eachother up.

The descriptions given by these participants aligned with the other discussions on competition, something that all participants had experienced. Competition was related to both drinking and sport. For instance, William describes competitive behaviour in drinking and rugby:

I guess like even in terms of rugby you want to win in terms of like drinking it is sort of like who can like drink the most (laughs) in a short period of time and sort of get through it. 
Most participants, like William, referred to either drinking or sport in their initial discussion of competition. However, it was also acknowledged that competition is prevalent across other activities:

So when I say competition I mean it doesn't really matter what the activity ... so if that's an intellectual pursuit if that's playing chess doing sailing if that's doing rugby...they're all kind of competitive outlets where it's a way of gaining status. And I think men think about this a lot, and that what I mean about control it's like if I can have this status I have more control over my life compared to other men around me and that makes me feel more secure in my manhood.

Participants perceived competition to be associated with the broader concept of masculinity. In terms of mental wellbeing both enabling and constraining factors of competition were identified by participants. The positive factors identified by some participants was that competition can be a motivator for betterment:

You have got, you know, the good side of competition, which is, you know, I don't know, let's say it's for rugby, it's...again going back to rugby. Um, you know, you build up your own strengths, you practice, you get better, you know, you become a better person for it. A better person hypothetically.

Um, competitiveness can be good in terms of if you actually are successful in terms of being competitive, you can normally do quite well for yourself.

Five participants acknowledged this positive aspect of competition and that they enjoyed a level of competition, particularly when competing in sport. However, as the following participant highlights, competition inherently involves winning and losing. This has synergies with the idea of values, and what is good or bad. Self-reliance was something that was seen by participants as a value of "the average kiwi man", therefore there is the potentially that not showing self-reliance is synonymous with losing in a competitive stance. This idea was discussed by the following two participants:

Yeah maybe like that they just that competitiveness maybe plays a role in that so that they don't want to seem weak and stuff. So yeah, they don't talk to other people and let them know what's happening.

a lot of these activities are around competition and measuring up on each other uhm and because of that state I feel like you're constantly in a state of semi fear 
or anxiety about what other men think of you and because of that you're not really gonna be vulnerable with them.

Both participants discuss competition and the idea of mental health issues or mental vulnerability. They both describe how men wouldn't want to "seem" weak or are concerned with what other men think of them. The concept of emotional restraint because of this stigma leads to the final category in these findings.

\subsubsection{Devaluation of emotional expression}

In their description of the "kiwi bloke" Sim \& Thompson (2000, p.4) highlighted that there was constant "denial of pain" and "undermining of emotions". Each participant in this study addressed the theme of not showing emotions. It was the only category in this section that participants acknowledged had no potential positive effects on mental wellbeing:

You know, if you're actually not mentally tough and your pretending to, that's just the whole antithesis of what you want to be doing... So I feel like that one's pretty, yeah, I don't think there'd be a huge amount of benefit to the tough guy act.

Most participants discussed restraining emotion and vulnerability as something that was still an issue in New Zealand. Three participants explicitly related having to be a "tough guy" or "harden up" to New Zealand suicide rates. The following quote is one illustration:

I think it's just because of that stigma of, you know, just get on with it... I think that's really bad, and it's slowly getting better, but you know, looking at like the youth suicide rates and all that, it's just horrendous.

All participants acknowledged there was no benefit to emotional restraint, particularly when it came vulnerabilities, and given the discussions surrounding the prevalence of New Zealand's suicide rates it was interesting to hear participants' perspectives. The following participant provided his perspective on the complexity of this issue:

well cuz men don't, well not, I mean I'm stereotyping here but the majority of men don't wanna talk about stuff, just because you've got societal pressure to be the one that provides for your family and stuff like that, you've got the competition so you always wanna be the better man, just the stuff like "I don't wanna air my dirty laundry in public", it's my issue I'll deal with it, and the fact that we always think you can deal with it but maybe you can't. Like you're weak if you ask for help, there's a lot of stuff just like a combination. 
Tristan describes how men stereotypically “don't wanna talk about stuff”. In the context of this discussion he was referring to emotional or internal issues. He then subsequently highlights various reasons for why this might be the case, which interestingly encompasses many of the themes previously addressed throughout these findings. The following participant discusses how he feels that it is not just negative emotions that men are restricted from displaying:

I feel like I'm a person who gets really excited about things. Um, but that's not normal. Like amongst my male friends.....And it's not really a thing that is part of that male landscape or showing lots of joy, like just really, you know, unrestrained joy or excitement or, so it's almost like the emotional model of a man is very like in this middle, middle line without like going further on each side. It's very kind of muted.

Mike emphasises how his expression of excitement is not "normal" or part of that "male landscape". From his perspective, it is not just the expression of negative emotions that are restricted by a normalised model. He describes the emotional model of a man being "muted". This is a very interesting perspective because within the mental health literature and also popular media there is often a focus on the need to encourage men to open up when they are struggling or dealing with issues (Eisenberg et al., 2009; Seidler et al., 2018; Skerrett, 2019). There is a lack of academic literature which looks specifically at the possible restraint of displaying excitability or joy. The following participants' discussion provides an interesting parallel to Mike's statement:

It would be surprising for me to see one of my friends get super emotional all the time... or be, you know, very quick to, you know, have like a super strong emotional response to something.

Tony places emphasis on how it would be out of the ordinary for his friends to display strong emotions, reinforcing what Mike was describing about his own personal experience. The following two participants make the association between certain groups and the inability to show emotion:

They probably wouldn't understand, a lot of sports people...you get the kind of people lumped together there where nobody really knows, would know how to deal with it...a lot of them their thing is even if they're struggling with it, they 
probably have the mentality that, you know, it'll fix itself and so they're not going to be great help for somebody.

I don't think vulnerability is welcomed in that, in that group for sure...I think they get scared of seeming weak in those groups.

Here, Daniel and Harry discuss sports groups they had previously described as being the popular or dominant groups of men. From Harry's perspective, he feels as though a group of sports people wouldn't understand if someone were to be struggling. Whilst Daniel describes vulnerability as not being welcomed. However, they are both also implicitly acknowledging that people within those groups may also have their issues. Harry says that if they are struggling, they probably have an "it'll fix itself" mentality and Daniel discusses how within the group these guys might fear seeming weak. Which leads into a very interesting finding surrounding the concept of a "double bind" when it comes to displaying emotions:

Yeah. So it's kind of do or you don't in the sense that, um, if you do tell them you're probably going to get ostracized and that'll make you feel worse. But if you don't, for one, you'll always have the fear that you're going to get ostracized from the group. Yeah. And then you have to deal with what you're going through anyway.

So it's kind of a conflict between the two and there's this huge pressure to be that person, that strong guy but at the same time it's not really who I am as a human being... But if I wanna be this sort of sensitive emotional boy I can't really do that without feeling very vulnerable, so I'm pulled between the two.

Through these discussions we can see a difficult tension between the construction of masculinity and mental wellbeing. The participants illustrate that to align yourself with the dominant group means it may be necessary to display strength and restrict vulnerability; however, if you chose to share your sensitivity or issues there may be a risk or even just a perceived risk of being ostracized which can exacerbate an already present issue. Whilst some participants acknowledged that the stigma surrounding emotional expression was potentially slowly going away, most participants acknowledged that it was still very much an issue. The following participant made an interesting connection to emotional displays being redirected:

Not being allowed to cry and not being allowed to say that I'm afraid is... that's the worst thing you can do for a young man. I think like who's going through 
something hard and it just bottles up and it comes out with ripping on other males or it comes out as treating females poorly or like it comes out as being violent when you're drunk.

This participant provides insight into his perspective on how emotions such as crying, and fear can be redirected. The use of alcohol as a potential route for intimacy or emotional venting was discussed by seven participants in this study. The following participant discusses drinking and emotional intimacy:

I think it's a real, it's a real avenue for emotional intimacy without needing to like be fully cognisant about it. So it's the ability to have those like, "Oh, I love you man" things. I mean, you have a hangover, you wake up, then you'd go back to your normal, Kiwi bloke life. um, so it's almost like an outlet for, for, for this intimacy, but obviously it's not the outlet you would want, but like, yeah. Yeah. It's kind of strange in that way.

This participant is not discussing the use of alcohol to talk about issues or negative emotions, instead he describes how drinking can enable men to express their feelings towards one another. A recent study on the effect of alcohol on emotion in social drinkers (Sayette, 2017) found that alcohol had a different impact on a group of male social drinkers than that of females. Male groups were found to receive greater alcohol reward, so their benefit of emotional expression through the consumption of alcohol was greater than that of females. This is perhaps because the expression of emotion when combined with heavy intoxication is socially acceptable. The lowering of inhibitions during a drunken state may enable the communication of emotions not otherwise expressed between men, for instance:

uhm men don't share feelings with each other, not really, not unless you're both wasted at a party, and someone's like "aw my girlfriends gonna leave me" ya know, when they finally feel vulnerable enough to share it with their friend because they're both in that kind of happy state together.

This statement relates to drinking as a positive social lubricant. However, these participants are emphasising the negative implications of drinking being one of the only avenues for emotional expression. The following participant provided a reason for why, from his perspective, men only share emotions through the practice of drinking:

cuz they don't know how to deal with it in any other way, and there hasn't really been a template or format of teaching them how to deal with stuff. 


\subsection{Closing statement}

The findings from this study provide insight into the complex relationship between masculinity, mental wellbeing and the student experience. Drinking and physicality were two of the most prevalent themes that were identified, both of which played a role in social bonding and competition which were perceived as key factors which effect mental wellbeing. The following chapter will discuss these findings in relation to relevant literature. 


\section{Chapter 5}

\section{Discussion}

This chapter provides a synthesis and critical analysis of the findings presented in the previous chapter. Four overarching themes were found within this study and respective subthemes were identified. Together, they offer a rich perspective on the topic. The section discusses specific findings from this study with the wider literature. This chapter draws strands from the findings and theorizes them in greater depth using the works of R.W Connell (Connell, 1985, 1997, 2005; Connell \& Messerschmidt, 2005) and Erving Goffman (1976, 1963, 1959, 1967).

\subsection{The persistence of a cultural script for New Zealand men}

A cultural script consists of collective elements such as norms, values and practices within a group. The idea of a script in social interaction was central to Goffman's (1959) work on dramaturgical sociology. It provides guidelines for 'performative' action or behaviour that is deemed appropriate. This does not mean it guides individual behaviour, rather it provides a "backdrop" for how individuals position their acts in social groupings.

Examining consistencies that shape masculinity across history in specific cultural contexts offers insight into how practices and values may have changed over time. This study identified elements of masculinity in New Zealand that have been discussed as hegemonic practices in prior literature. Whilst the "average" does not necessarily constitute the hegemonic, when perceptions of the typical are combined with perceived expectation and/or idealised representation (i.e. heroes or role models) the hegemonic form of masculinity may be ascertained. Hegemonic masculinity does not need to be practiced or embodied by most men (Connell \& Messerschmidt, 2005), as it is culturally exalted, its existence can inform the practices and values of complicit and marginalised masculinities.

The prominent "ingredients" of the "kiwi bloke" identified by participants, such as drinking and sport, have distinct synergies with accounts made by two New Zealand authors: Gwendoline Smith (1990) and Michael King (1988). Interestingly, these accounts were made 30 and 60 years ago, respectively. In the following section, the insights from these works are juxtaposed against the findings in this study. Subsequently the "underlying mechanisms" that reinforce a persistence of the cultural script are discussed. Elements of the cultural script will then also be discussed in relation to the role of the male student and the "life course" of men. 


\subsubsection{The "kiwi bloke": a cultural echo or a relived experience?}

Literature surrounding the "kiwi bloke" and masculinity in New Zealand highlights the prevalence of sport and drinking (Joseph \& Falcous, 2017; Willot \& Lyons, 2012). This nexus between men, alcohol and sport has a deeply intertwined history that can be observed in many countries (Wenner \& Jackson, 2009). Interestingly, this study found that male students also placed an emphasis on the practices of sport and drinking. These two factors eclipsed many areas of the interviews and were not just discussed in response to the practices of the "average kiwi man".

Participants discussed physicality and sport in relation to high school experiences, the description of a "do it yourself" kind of man, and inherently through the common reference to Richie McCaw and the All Blacks. This latter sub theme regarding Richie McCaw and the All Blacks was an interesting finding because it relates quite distinctly to a discussion by Connell (2000). In this discussion, Connell explains that for hegemonic masculinity to be culturally exalted, then this pattern of masculinity must have those who are celebrated as heroes. It was interesting to find higher education students commonly referring to an All Black as an embodiment or role model of masculinity in New Zealand, given the contrast between a successful rugby player and a successful student. When this finding is combined with the emphasis that participants placed on the importance of sports in high school it suggests that sport is not just a practice but that it is of value in New Zealand. It raises a question as to whether it shapes the experience of male students as they move into higher education, a context where physicality is not necessarily emphasised or centrally celebrated.

Drinking is something that was also discussed in various areas of the interviews. It was discussed in relation to the "average kiwi man" but also, distinct from sport, it was highlighted as a prevalent practice at university. Participants also highlighted New Zealand's "binge-drinking culture". The practice of binge drinking specifically denotes a type of drinking behaviour that is competitive and aligns with the literature on the masculine orientation of "being able to handle your drinks" (Penwarden, 2010; Courtenay, 2000). Wenner and Jackson (2009) describe how those who drink more are regarded as "manly" whilst those who drink less run the risk of being chastised. Therefore, there is a problematic "compounding" of factors which likely effect the male student experience e.g. the expectation to drink at university, drinking to reinforce masculinity and adopting "bingedrinking" culture that is part of the broader social context. 
As well as drinking and sport, there were several other themes that emerged from this study which aligned with Sim \& Thomsons (2000, p.4) description of the kiwi bloke; "men are characteristically loyal to their mates, constantly exchanging banter...but most importantly displaying masculine feats of strength, courage and competency with machinery, and denial of pain. There is always a constant undermining of emotions". This statement holistically represents the themes of loyalty, banter and the undermining of emotions.

The title of this section posed a question: "A cultural echo or a relived experience?" Some participants in this study highlighted that they felt the "kiwi bloke" stereotype was fading. However, upon further research there were some distinct similarities between the discussion of participants in this study and accounts given by two New Zealand authors. The first account was given by Michael King in his 1988 book "One of the boys? Changing views of masculinity in New Zealand”. King describes his experience growing up in New Zealand in the 1960/70's:

"I had an ambivalent relationship with rugby, as I did with other major ingredients of male culture, alcohol and sex...I used the time allocated to sport to read poetry and novels. This cast me as an outsider...I managed to avoid intensive personal encounters with alcohol- that other ingredient of manliness- until university."

His description has many resonances with accounts given by participants in this current study, almost 60 years later. Particularly how he ended up participating in rugby to avoid being excluded from the "easy camaraderie" that young men would display on and off the field. There was a similar notion of loyalty described by some participants in this study, which mostly surrounded physical situations. One participant also relevantly described how this sense of "teamwork" was only for the boys who "made it into that model".

Another link between King's description and this study is how he managed to avoid drinking until university. He subsequently details how he began drinking in university to "fit in", his group labelling those who did not drink as "earnest and boring". This ties into participants" descriptions of the importance of drinking as a social bonding activity in their first years of university, but also their perception that not participating in drinking as a social activity could lead to ostracization.

In 1990, Auckland psychologist Gwendoline Smith published her book "Will the real Mr New Zealand please stand up?" The book is a collection of accounts from various men around New Zealand with a core focus on the emotional nature of men. Again, there are some 
surprising similarities between the observations and accounts given in the book and the participants' discussions in this study. She is surprised that a man describes New Zealand men being into "rugby, racing and beer" posing the question, "Did he really think this stereotype was still alive and well in New Zealand? If so, why did it continue to flourish?" (p. 187). Another man discusses how he would not share his problems with other men, however he believed in the next decade it would be a lot easier: "What happened in the seventies doesn't happen in the eighties. We are just a little bit slow at waking up though, aren't we?" (p.200).

This study does not provide a definitive measure of the extent to which "rugby, racing and beer" is an ongoing stereotype, nor can it provide a measure of how different the participants current experiences compare to the account provided by Smith and King, 30 and 60 years ago. However, the similarities across the sample of male students in this study highlights that some of the same "masculine ingredients" are still well versed. Most participants also acknowledged that they felt compelled to act in accordance with the practices and values that they were discussing. They perceived that doing otherwise may result in being "pushed out".

\subsubsection{The "underlying mechanisms"}

Findings from this study, alongside relevant literature, provide some insight into possible underlying mechanisms that reinforce practices within the cultural script, particularly sport and alcohol. The term "underlying mechanisms" is used because these are elements of the findings that were not necessarily explicitly described by participants. However, from a collective and holistic viewpoint there are interconnections that can be made between themes in this study and relevant literature. It is also fittingly descriptive of the way in which these mechanisms are subconscious, ambiguous and complex.

Competition was discussed by several participants. It was described as something that was "just there" - or as one participant stated, "this stupid thing that happens". Several themes identified in this study (such as sport, drinking and banter) all had competitive elements. The concepts of masculinity and competition have a complex relationship. It is something that fuels many activities within a man's life, in an attempt to fill a hypermasculine script. In Goffman's (1967) essay, "Where the action is", he described how scenes of action allow for the portrayal of character, particularly an individual's degree of composure. He describes composure as the ability to display a sense of calmness and control of emotion. Therefore, sites of masculine action provide a way to "measure up", they are competitions to determine 
who has the most "character" and therefore where they are situated in the internal and external hierarchies of male groups.

During discussions with participants, the terms "us" and "them" were used when describing elements of group dynamics. The language that they were using seemingly divided participants into the parts of the "average" male with which they identified. However, there was also the explicit recognition of group dynamics, for instance; popular groups in high school, being welcomed into a group of men, and the idea of being ostracized. Intermale hierarchies and group formation is another mechanism that is implicit in the performance of masculinity. It is discussed significantly by Connell (2005) through the concept of masculinities. Male orientated practices that are often competitive sites enable the construction of "in" and "out" groups as well as a hierarchy within groups. This is easily observed in male dominated areas where physicality is a focus e.g. sports groups, the military and gangs (Donovan, 2012). It is also observable in areas that are restricted to men, such as all-boys' schools and fraternities.

When competitiveness is combined with group dynamics people will seek to avoid anything that will place them at the "bottom of a hierarchy" or socially ostracized altogether. Therefore, what is perceived to be weak or undesirable, even if it is perhaps necessary or logical, might be denied. As Courtenay (2000) describes, this is made clear when a man positively states that "he hasn't been to a doctor in years", which simultaneously puts his health at risk but also displays his masculinity. There is an unfortunate irony: risk-taking behaviour is commonly attributed to men, yet the risk of appearing vulnerable or weak is not one that is widely viewed as worth taking.

Participants in this study discussed the restriction of emotion and vulnerability as a continuing issue in New Zealand, and they all acknowledged that it was not beneficial for mental wellbeing. Some participants also discussed the association between emotional restraint and New Zealand's male suicide rates, which have become of increasing concern for New Zealand and other OECD countries (Pitman, 2012). Despite an increase in awareness campaigns, there is still a notable lack of help-seeking from men and an increase in young male suicides rates. Even when men overcome barriers to help seeking, there is a common issue surrounding continued engagement with health services (Seidler et al., 2018). Whilst awareness and education are important in reducing initial stigmatization, there is perhaps 
also a need to mediate the relationship between the underlying mechanisms of competition and social-group formation amongst males.

\subsubsection{The impacts of the cultural script on male students in higher education}

It is important to consider the implications of the findings for male students in higher education. Goffman's (1959) concept of dramaturgy is particularly helpful here, especially when supported with West and Zimmerman's (1987) concept of masculinity as a "master status". Dramaturgy posits that we are all actors on a stage, and we will alter our performances based on the audience watching. For example, a male student will have different sets of "mini scripts" dependent on the situation he is in and who is present. At university he may present differently in front of a professor, other students, friends and family. However, associated idealizations of masculinity within broader culture can have an influence on all of these "mini scripts". Earlier, the literature review highlighted that, historically, the "kiwi" bloke raised questions about the masculinity of "intellectuals" (Bannister, 2005). Intellectual men felt a pressure to emulate dominant masculinity, proving their solidarity with “ordinary blokes". Willot \& Lyons (2012, p.332) also discuss how men at university in America have been found to use public drinking to demonstrate their "hegemonic status to others" as an alternative to academic pursuits.

Within this study participants identified the prevalence of drinking at university; however, some participants also acknowledged that being an "intellectual", or just focusing on academic activities, did not constitute being "manly". There were multiple layers to this acknowledgement, e.g. reading as a "segregating" activity, academic work as not conducive to male bonding, and adopting other practices or finding friend groups that can reinforce masculinity. There was also the mention of "the lads and the boys" which was primarily used as a descriptor by participants for boys their age. It defined the "in group", or the hegemonic "mini script", that was prevalent for a young male's initial university experience. This experience is one that emphasises the expectation to drink and party, and the literature has associated it with the rejection of academic hard work (Jackson, 2010).

Jackson \& Dempster (2009) observed that the association between academic hard work and "uncool" masculinity encourages male students in higher education to present themselves as effortless achievers. This can produce a difficult contradictory complex for some male students who try to balance masculine portrayal but simultaneously avoid failure. For those who are unable to achieve this balance, there is the risk of a cyclical relationship between 
failure, self-worth and avoidance of hard work. These dynamics invite us to consider the shift in achievement between males and females at tertiary institutions in New Zealand over the last two decades. There are now significantly more female students enrolled at all levels of tertiary education and they are also more likely to complete their qualifications (Irwin, 2011).

\subsubsection{The convergence of the life-course and the "masculine script"}

The "life-course" was a theme that was identified through the implicit tendency of participants to identify prevalent practices and values that were associated with life stages. The three relative stages were high school years, current experiences and future expectations. For instance, sport and social groups were discussed in relation to participants' past experiences and high school. Whilst the "kiwi bloke" and the "kiwi dream" were discussed in terms of older males or future pursuits. Comparatively there was limited discussion surrounding masculinity in relation to the participants' age group, other than the concept of the "lads and the boys".

Values were difficult for participants to discuss in comparison to practices. Whilst values can be generally harder to identify, they can also be linked to expectation. Therefore, a place such as high school which produces stringent expectations of "popular" social hierarchies, understanding what is "good" and desired is clear. Societal expectations surrounding what you should be achieving when you are older may also be easy to identify, i.e. a stable house, income and a family. Comparatively, in the stage in between, where one transitions from high school into the adult world, the formation of your own values begins to take place. This is a stage where independence and the "forging of your own path" is emphasized. It involves the consolidation of what an individual has learned during adolescence with what is expected of them in the future.

Studies which analyze the progression of masculinity through the life course are very limited (Spector-Mersel, 2006). However, there is perhaps an increasing need to look at this transition phase (approx. 18-24 years old) due to the considerable change in expectations of young men over the past few decades, specifically in the west. The theory of "emerging adulthood" suggests that the age group of 18-29 years is a new and distinct period of development (Tanner \& Arnett, 2011). It is characterized by "pushing back" the entry into stable adult roles. There are multiple negative and positive consequences associated with this 'new' developmental phase that could have an influence on mental wellbeing. There is also a paradoxical pattern for mental wellbeing during this phase. On the one hand, there is an 
experience of instability and a high risk of mental illness in comparison to other age cohorts (Tanner \& Arnett, 2011); whilst on the other hand, there is significant rise in factors associated with increased mental wellbeing, such as increased perceived social support, selfesteem and resilience consolidation (Tanner \& Arnett, 2011).

The period of emerging adulthood presents an instability for the monitoring of mental wellbeing from both research and practice perspectives. For young males the confusion of this phase may possibly become compounded as traditional values associated with the life path for men are altered. For instance, the expectation for men to be the sole provider for a household has altered as more women have entered the workforce. In many parts of the world, political and cultural forces have been moving towards gender equality in multiple areas of life. Whilst this brings about positive effects, it causes change to traditional expectations and roles.

Whilst traditional roles and norms were perhaps negatively restricting, they simultaneously provided structure and a "pathway" of values to adhere to. This central pathway likely contributed to individual and communal directions of values, helping to facilitate belonging through communal and group identity. Whilst the continuation of "masculine ingredients" has been discussed, this continuation does not necessarily mean that the power and positioning of these practices and values in broader society remain consistent. Historically, the markers to manhood were restrictive yet clear, and they gave direction to young men. It is entirely possible we are on the precipice of large-scale conceptualizations of masculinity being reverted to the individual. This may provide a great deal of opportunity and exploration for young men at the individual level; however, simultaneously it may be restrictive due to the added complexity of identifying positive values and future goals.

\subsection{Mental Wellbeing}

The following section is separate into four parts. It integrates strands from the findings, as well as some previous discussion points to further theorize the enabling and constraining effects of masculinity on mental wellbeing.

\subsubsection{The complexities of male bonding}

One of the aims and sub-questions of this research was to explore the ways in which perspectives on masculinity might enable constructive views on mental wellbeing. This area of research in prior literature was limited. It may be due to the rise of the term "toxic masculinity" and its focus on the constraining factors associated with masculinity. 
In the present study, the primary theme that emerged as a positive facet to the practices and values was their facilitation of bonding. The formation of positive relationships and strong support systems are particularly important for mental wellbeing (Barden \& Caleb, 2019). Close friendships can offer a sense of validation and self-worth, offsetting negative implications of any transition and change. Nicole Way (2011) and her research team conducted multiple interviews with young men and found that the boys saw their male friendships as essential components to their health. However, the way in which male friendships are displayed has a strong tie to the cultural construction of masculinity in the west. The proclamation of intimacy and connection between male friends is evident in the cultures of southern Ghana, Cameroon, Native American tribes, and until recently China and the Middle East (Way, 2011). In contrast, cultures which have seen influence from America depict males as lacking emotional skills and intimate ties to their friends.

There were two sides to the discussions on male friendships provided by the participants in this study. For instance, there were those who described bonding through adversity during team sport or perhaps risky drinking behaviour. On the other hand, there were some participants who described the friendships "within the script" as being only surface level. One specific participant described his friendship group as being very close and intimate, but he did not think that this was usual or average. This disparity is perhaps due to the implicit and complex way in which emotional connections are established in male friendships, especially the male friendships that are formed whilst operating within a masculine script where outward displays of intimacy and vulnerability are uncommon.

Easthope (1992) discusses how banter has a dual function in establishing intimacy whilst appearing to outwardly deny it. From the outside banter is aggressive and involves the often egotistical and underhanded remarks against another person. However, within a friendship the performance of banter involves an "intimate and personal understanding of the person who is the butt of the attack" (Easthope, 1992, p.88). Easthope describes the use of alcohol as having a similar function as the inebriation makes permissible displays of emotion or intimacy that would otherwise be chastised. This same "hidden intimacy" is discussed by Smith (1990) and Wenner \& Jackson (2009). The latter describes how men often connote rather than emote intimacy, through common interests or doing things together.

Therefore, sport or physical activity, banter and heavy drinking are perhaps sites to which male bonding can be established. There are certain questions that arise from this arrangement, 
such as whether it is necessarily negative. Also, whether it is entirely a product of a fear of intimacy. It also has certain implications for the wellbeing of male students in higher education. Belonging and engagement has become a core focus of student mental wellbeing initiatives in recent years (Thomas, 2012; Masika \& Jones, 2016).

\subsubsection{Male bonding in the context of higher education}

Establishing a sense of belonging within the higher education environment is particularly important for first year students. It is often the point to which the largest number of changes are taking place, including moving away from family, establishing new routines and creating new social bonds. A study conducted in New Zealand in 2011 showed that young males have a greater struggle with transition to university than their female counter parts (Irwin, 2011). The male students perceived that they had greater difficulty in establishing learning relationships and social contacts due to masculine self-image. An interesting finding in this study was that some participants suggested a need for more social initiatives that involved physical activity, because that was a way in which they could initiate social contact. They also were five times more likely than females to list the opportunity to drink and party as a highlight of university.

Whilst most participants within this current study acknowledged negative implications of drinking, some also described the pressure to drink during their earlier years and how it was sometimes their only means of socialisation. The emphasis on the importance of drinking during the initial years of university is consistent with studies conducted in the UK and US which showed consumption peaks before the age of 21 (Bewick et al., 2008). Another study has shown that whilst drinking was not related to males' general levels of satisfaction, heavy drinking was related to higher levels of social satisfaction (Murphy et al., 2005). It was suggested that men may be particularly dependent on the social benefit of drinking. In response to this issue, a recent initiative at Otago university called, "Men in Med", was created to provide male medical students with a way to socialise without drinking (Hall, 2018).

\subsubsection{The ambiguous line between healthy and toxic}

Whilst this research explored both enabling and constraining factors of perceptions on masculinity, the findings from this study highlight that the practices and values identified can be simultaneously positive and negative. A central premise was that factors such as competition, banter, drinking and sport are fine for some; however, they pose the risk of 
being detrimental. One participant highlighted that it was not necessarily the practices that were an issue for mental wellbeing, it was the behaviour and attitudes within the practices that held the potential to be problematic. The problematic behaviour possibly takes place at points of tension within the script when masculinity needs to be negotiated, which can result in bringing others down or alternatively risking one's own wellbeing to prove "toughness" (Courtenay, 2000).

The literature review highlighted that traditional masculinity is becoming increasingly intertwined with negative practices such as violence, crime and sexist behaviour (Connell \& Messerschmidt, 2005; Beynon, 2001). This has given rise to the term "toxic masculinity" which is increasingly becoming synonymous with hegemonic masculinity. However, as outlined by Lomas (2013) hegemonic masculinity maintains its position through a certain degree of consent and participation, therefore it must encompass some benign or positive characteristics. The findings from this study reinforced the existence of benign characteristics through the differing perspectives given by participants surrounding "masculine ingredients". However, there is a potential difficulty in navigating the fact that masculine practices and values can be simultaneously "good for some and not for others". The following statements from participants are illustrations of this dynamic;

sometimes feelings can get hurt

sometimes some guys get it more often than others

But some people, I can understand some people don't have the courage or just don't feel comfortable

The statements highlight the ambiguity of "sometimes" occurrences and that "some" individuals may feel affected by certain practices or behaviours whilst others view it as unproblematic. The line which determines the point where practices and values may become toxic can be difficult to determine. A micro-level example of this difficulty could be observed through the subtle distinction between malicious intent and bonding during banter. The participants that discussed the practice of "banter", or "rip culture", highlighted how it has the potential to be damaging, particularly for the receiver. As previously discussed, banter involves an intimate knowledge of the person to which the joke is directed to. There is a discreet and ambiguous line between bullying and banter, sometimes with the latter being used as a disguise for the intentional verbal harm of another. 
Practices can also become toxic at the macro-social level i.e. institutional or national. This was particularly clear through participants descriptions of New Zealand's "binge drinking culture". Many participants acknowledged the negative implications of New Zealand's drinking culture, referring to things such as "crate day". This day has become somewhat of a tradition in New Zealand and involves an individual attempting to consuming an entire crate of beer.

As previously discussed, participants also highlighted the prevalence of drinking in the university environment; particularly during the early years of their university experience. However, alcohol is used within almost every culture worldwide to facilitate celebration and as an accompaniment to positive social situations. Again, there is a balance which must be ascertained between healthy and "over-doing it". In certain situations, it is clear when drinking is being misused, but facilitating a healthy relationship with drinking is something that is difficult to encourage when dealing with broad attitudes towards the practice at group and national levels.

Competition and self-reliance were two more themes that can also be viewed as "double edged swords". For instance, there were participants who acknowledged the good side to competition such as the ability to better yourself as an individual. Others highlighted that competition causes pressure and compounds the ability to show any kind of weakness or vulnerability.

\subsubsection{Stigma and the double bind}

The literature review highlighted that men are often viewed as "damaged and damage doing" (Lomas, 2013). The concept of a possible dual stigma was discussed, whereby men may be stigmatized for being overtly masculine and displaying "toxic traits" whilst also not being able to show vulnerability or a struggle. A "double-bind", or a "damned if do, or damned if you don't situation", was recognised by participants in this study. Participants described how hiding internal "weakness" or struggle is not preferable, but neither is the possibility of appearing as "other" or being subject to stigmatisation. For men who identify with the dominant masculine script but suffer through a period of diminished mental wellbeing, there is the possibility of an existential crisis of sorts.

Multiple participants acknowledged this problematic relationship between perceptions of masculinity and mental wellbeing. Despite initiatives to increase awareness (Skerrett, 2019; Marvelly, 2019), the difficulty in being emotionally vulnerable or expressive was described 
by participants as being a current and prevalent issue. One participant highlighted that "bottling it up" often comes out as "ripping on other males" or "treating females poorly" or "being violent when you're drunk". Courtenay (2000) suggests that when men are denied access to what is deemed contingent with social power, they tend to seek other resources for constructing their masculinity. Therefore, if they feel socially "unworthy" or that how they are feeling is not contingent with what is expected of them then they will often resort to alternative means of coping rather than seeking further help or expressing their feelings outwardly. This also aligns with Brownhill and colleagues (2005) concept of the "big build". Interestingly, there was a participant who discussed the difficulty in displaying positive emotions such as excitability, which often meant he was labelled as 'gay'. Connell (2005) has noted that historically subordinate masculinities that are labelled "other" are often linked with the feminine or homosexual. This makes sure there is separation between what constitutes "manly" and what doesn't. This finding stood out because both academic literature and broader mental health initiatives tend to be aimed at getting men to speak out when they are struggling (Eisenberg et al., 2009; Seidler et al., 2018; Skerrett, 2019). However, the restriction of joy and excitability may be just as constraining as the inability to show pain and sadness.

The cultural script is inherently restrictive and provides a "guideline" for stigma. This restriction may lead to diminished mental wellbeing. However, it is important to note that two participants in this study reported that they felt comfortable within multiple different social groups. When asked why this was the case, both described "confidence" as an important factor. In some ways, these participants seemed to be social chameleons, with an ability to move within scripts with relative ease. Confidence is an interesting theme that aligns with previous studies by Harris (2010) \& Hong (2000) and which would be a good area to consider for future research and practice. 


\section{Chapter 6}

\section{Conclusion}

This research is located at the intersection of the literatures on masculinity, mental wellbeing and higher education. It addressed the following research question and two respective subquestions:

How do perceptions of masculinity shape the ways in which male students perceive mental wellbeing?

- How does masculinity enable constructive views on mental wellbeing?

- How does masculinity constrain views on mental wellbeing?

The literature highlights the complex ways in which masculinity may affect the mental wellbeing of men. Traditional expectations associated with masculinity place emphasis on competition, achievement and strength (Howson, 2006). Subsequently these expectations may restrain factors which are conducive to mental wellbeing such as help-seeking and emotional expression. This restraint places men in a vulnerable position when it comes to dealing with diminished mental health. Concurrently, the literature highlights the prevalence of "toxic masculinity" which often associates masculinity with violence, sexism and risk taking (Connell \& Messerschmidt, 2005; Beynon, 2001). Therefore, masculinity and its effects on mental health creates a "catch-22" situation whereby men are often situated as both "damaged and damage doing" (Lomas, 2013).

This research explored perceptions of masculinity and mental wellbeing in the specific cultural context of New Zealand and higher education. Erving Goffman (1976,1959) and R.W Connell (Connell, 1985, 1997, 2005; Connell \& Messerschmidt, 2005) were the leading theorists that underpinned this study. Their perspectives emphasise the multiplicities of masculinities and, in Goffman's case, the multiplicities of roles we play in our every-day lives. The acknowledgement of the fluidity of masculinity lent itself to the culture studies approach adopted in this study. The "kiwi bloke" or "southern man" is New Zealand's version of hegemonic masculinity which stems from a unique cultural identity marked by colonialism and a remote environment (Sim \& Thomson, 2000; Joseph \& Falcous, 2017). The effects of hegemonic masculinity on mental wellbeing in New Zealand is discussed 
sparingly within academic literature. Even more so, there was a significant gap in the literature which explored this topic from the perspective of male university students.

Higher education institutions have been increasingly concerned with the ways in which they can provide their students with support (Baik et al., 2019; Storrie et al., 2010). The unfamiliar transitionary period from home life to higher education presents many challenges for young people including establishing new social networks and life skills (Barden \& Caleb, 2019). It is important that we understand the diverse ways in which academic life may interact with student's mental wellbeing in order to develop effective solutions and practices. This research addressed a gap in the literature by exploring the ways in which male students in New Zealand perceive masculinity and mental wellbeing. The following sections detail the key findings from the study and provide a summary of insights. The chapter concludes with possible limitations as well as suggestions for future research and practice.

\subsection{Summary of findings}

Themes identified through the analysis were compiled into four over-arching groups and respective sub-themes. Together they provide insight into the complex relationship between masculinity, mental wellbeing and the student experience. The findings from this study make several original contributions to the literature. Firstly, the findings highlight the prevalence of physicality and drinking as centralised components of a continuing "cultural script" for male students in New Zealand. This finding supports academic literature which also lists physicality and drinking as prominent identifiers of the "kiwi bloke" (Joseph \& Falcous, 2017; Willot \& Lyons, 2012). However, there were several interesting findings that provide insight into how these practices are currently relevant to young men in New Zealand.

For instance, Richie McCaw and the All Blacks were discussed by most participants in this study, which provides a modern-day representation of the "ideal male figure" in New Zealand. The idolization of rugby players by young men, or at least the recognition of such an idolization, reinforces the value of physicality and strength. The participants in this study also discussed the prevalence of sport at high school. Playing sport was something that participants perceived to determine popularity and entrance into the "in group". The emphasis on sport in high school, combined with the idolization of rugby players, suggests that physical pursuits are not simply expected practices, but that physicality is a valued enactment of masculinity in New Zealand. 
Given the importance of sport during the high school period it was interesting to see how physicality would be relevant in a higher education environment, where mental performance is prioritised over physical. Participants discussed how drinking became a significant part of their university experience, particularly in the first year. They perceived drinking alcohol to be a useful "social lubricant" that enabled the formation of friendships. However, in turn, it means that choosing not to consume alcohol could lead to social isolation, especially when forming bonds with other male students. Participants' descriptions suggested that drinking behaviour effectively replaces the values of sport and physicality in university.

These findings highlight the instrumentality of physicality and drinking in outlining the expected masculine displays in different educational contexts and periods of life. Their ability to determine social groupings and ease of socialization emphasizes that these are valued practices that continue to influence young men in New Zealand. In contrast, some participants discussed how a focus on academia was not something they perceived to be masculine. This was in reference to both academic practices such as reading, and the need to pursue other pursuits in order to feel masculine. Banner (2005) has highlighted that, historically, the masculinity of intellectuals in New Zealand has been questioned. However, the relationship between academia and masculinity is not something that has been explored in-depth. The findings from this study present a potential avenue for further research which would provide a more detailed account of how academia itself is viewed by male students.

The findings also provided various insights into the complex ways in which masculine practices and values may affect mental wellbeing. This study explored how the practices and values that were identified could both enable and constrain mental wellbeing. Participants discussed how shared activities and expectations can facilitate social bonding, which is often important for feelings of validation, self-worth and support. Therefore, participating in physical activities or drinking provides avenues for the establishment of male friendships. However, there were some participants who described how the practices and values "within the script" also created the possibility of social isolation. It was highlighted that going against the majority can make you feel alienated, unaccepted and restrict possibilities for making social connections.

Finally, one of the most explicit constraining effects that participants identified was the restraint of emotions. Every participant in this study acknowledged that there was no 
beneficial aspect to 'not being able to show your emotions', yet they felt that restraining feelings was still something which was expected of men in New Zealand. It was described as a "damned if you do, damned if you don't" situation. The pressure to be a "strong guy" or "tough it out" means concealing your emotions and perhaps not getting the support that is needed; yet, if you choose to express your feelings, you risk being met with ostracization. This finding is important as it highlights that the traditional "denial of pain" and "undermining of emotions" that is associated with the kiwi bloke (Sim \& Thomson, 2000) is a continuing rhetoric. Even though the context of higher education in 2019 is far removed from the rural environment of the traditional "kiwi bloke", the emphasis on mental strength still resonates with young male students.

\subsection{Summary of insights}

The findings from this study were theorized in the Discussion to draw further insight and comparison with the literature. The accounts given by participants showed several similarities with two New Zealand authors. The first author was Michael King (1988) who describes his experience as a young male in New Zealand in the 1960/70's. The second author was Gwendoline Smith (1990) who interviewed men across New Zealand in the 1990's to discover the "emotional nature" of men. Comparing findings from this study with the descriptions provided by King and Smith highlighted a consistency of "masculine ingredients" in which young men in New Zealand are still well versed. Whilst increasing urbanization and economic development in New Zealand has undoubtedly changed the way people live over the past sixty years, the extent to which the country's masculine culture has altered is unclear. This poses the question: are young men experiencing a "cultural echo" or a relived experience?

The Discussion also considered "underlying mechanisms" that lead to the persistence of a "cultural script". Participants discussed competition, banter and the restraint of emotion, which upon reflection, represented "micro practices" within the explicitly identified practices of physical activity and drinking. These "micro practices" are easily observable in areas where physicality is prioritised and in areas that are restricted to men (Donovan, 2012) e.g. the military, gangs, all-boys' schools and fraternities. Competition was one of the most prominent factors which seems to "fuel" the "tough guy" act but also determine a hierarchy of masculinity. Connell (2005) inherently refers to this hierarchy through the concept of masculinities where there are hegemonic, subordinate and marginalised forms of 
masculinity. Goffman (1976) also refers to sites of male action being a place where men are expected to "measure" up and determine their place in a social hierarchy.

Inherently the segregation of different groups means that there is a creation of "us vs them" which suggests a paradoxical situation for the facilitation of men's mental wellbeing. So, the interesting finding from this study is that masculine practices and values can be simultaneously "bad for some and good for others". Some participants highlighted that it was not necessarily practices, such as drinking and sport, that would constrain mental wellbeing, but more so the behaviours and attitudes that surround them.

\subsection{Limitations}

Due to the explorative nature of this study, it incorporated a relatively broad perspective on the intersections of New Zealand culture, masculinity, mental wellbeing and higher education. Therefore, the interplay between subcultures at a localised level was not specifically addressed. For instance, this research did not specifically represent various populations of male students, such as those from gender diverse backgrounds or ethnic minorities. Whilst all participants were born in New Zealand, only one student partially mentioned Māori, but did not specifically identify as Māori. No participants were asked for, nor discussed, their sexual or gendered orientation. Male students within such populations may have different perceptions on this topic.

The recruiting process may have influenced the data that was collected for this study. Participants were recruited through snowball and volunteer sampling. It is possible that some of the participants who volunteered had a unique interest in this topic. However, the snowball sampling method and incentive added (supermarket voucher) could have balanced this factor out, ensuring a range of motives for participation.

\subsection{Implications for future research}

Since the scope of this thesis was quite broad, it would be interesting to conduct a study across a range of demographics and compare the results to highlight differences in perceptions on this topic. This research may be specifically important for ethnic groups in New Zealand, especially indigenous Maori. There have been studies which examine cultural identity, psychological wellbeing and academic achievement for Māori university students (Gavala \& Flatt, 2005; Hunt et al., 2001); however, these studies involve specific academic disciplines and do not include a gendered perspective. There is sparse research that is 
conducted across tertiary organizations that explores perspectives on masculinity or mental wellbeing held by Māori and Pasifika students.

Another possible implication for future research would be the exploration of perceptions of academic hard work. The findings in this study suggest that male students do not regard academic hard work as "masculine". Whilst this perception may not be particularly harmful in and of itself, research has clearly shown a downward trend in male student achievement at the tertiary level (Irwin, 2011). The connection between masculinity and underachievement in New Zealand tertiary institutions is perhaps an area that requires further in-depth research. Future research could incorporate perspectives from male students who have "dropped out" of university during their undergraduate years, and their experiences since then.

Lastly, one of the core findings that emerged from this study was that of "underlying mechanisms" such as competition, and the establishment of "in versus out" groups in a male hierarchy. The sites of competition and the formation of social groups logically goes through a "reform" during the transitionary period from high school to university. Whilst most universities in New Zealand provide initial first year support to encourage students to establish social networks, there is a possibility to explore this transitionary period from a gendered perspective. Research on how different demographics of university students in New Zealand cope with the challenges during this transition could help to inform more effective support strategies for higher education institutions.

\subsection{Implications for practice}

The implications for future practice are mostly applicable to higher education institutions. The findings highlighted the importance of social bonding that masculine practices provide. Some examples of initiatives that already facilitate male bonding have been highlighted, such as "men's sheds" (Woosler, 2018) and the Otago program for "men in med" (Hall, 2018). The latter was created in order to provide an alternative to drinking that supports male medical students in forming friendships. The positive effects of this initiative are yet to be determined as the program is new. However, substitutes for the 'social glue' provided by alcohol should be considered by higher education institutions. In larger universities, it could be more challenging to create a "community" through which students establish a sense of belonging, so individual disciplines could consider increasing opportunities for engagement. 
Another implication for future practice would be to provide male students with a diverse range of role models. The students in this study discussed how many young men look up to rugby players or sportsmen. Having such a role model is not inherently negative; however, it creates a very homogenous view of what a successful man in New Zealand does, or how he looks. Social hierarchies are complex structures developed over the course of human history and it is perhaps Utopian to imagine that they could fade; however, promoting a diversity of masculinities in New Zealand could be a way to minimise the ways in which young men view themselves as different from, or "less" than, their peers.

\subsection{Concluding statement}

This study provides insight into the ways in which masculinity and mental wellbeing are perceived by male students in New Zealand's higher education institutions. The findings highlight the complex ways in which masculine practices and values may affect the mental wellbeing of male students. They also inform a current account of how masculinity is perceived in the cultural context of New Zealand in 2019. Ongoing exploration and questioning of the salient role of masculinity is important in order to understand its effects on mental wellbeing and to fuel its positive progress in as many ways as possible. I leave the closing words in this thesis to Erving Goffman (1963):

The normal and the stigmatized are not persons but rather perspectives. (p. 138) 


\section{References}

Adams, J. E. (2018). Dandies and desert saints: Styles of Victorian masculinity. New York: Cornell University Press.

Adler, J. M., \& Hershfield, H. E. (2012). Mixed emotional experience is associated with and precedes improvements in psychological well-being. PLOS One, 7(4), e35633.

Agger, B. (2014). Cultural studies as critical theory. New York: Spon Press.

Alasuutari, P. (1996). Theorizing in qualitative research: A cultural studies perspective. Qualitative Inquiry, 2(4), 371-384.

Alvesson, M. (2012). Understanding organizational culture. Los Angeles, USA: Sage.

Ando, H., Cousins, R., \& Young, C. (2014). Achieving saturation in thematic analysis: Development and refinement of a codebook. Comprehensive Psychology, 3, 03-CP.

Baik, C., Larcombe, W., \& Brooker, A. (2019). How universities can enhance student mental wellbeing: the student perspective. Higher Education Research \& Development, 1-14.

Baldwin, J. R., Faulkner, S. L., Hecht, M. L., \& Lindsley, S. L. (Eds.). (2006). Redefining culture: Perspectives across the disciplines. New Jersey: Laurence Erlbaum Associates, Inc.

Bannister, M. (2005). Kiwi blokes: Recontextualising white New Zealand masculinities in a global setting. Genders OnLine.

Barden, N., \& Caleb, R. (Eds.). (2019). Student Mental Health and Wellbeing in Higher Education: A practical guide. Los Angeles, USA: SAGE Publications Limited.

Barriball, K. L., \& While, A. (1994). Collecting data using a semi-structured interview: a discussion paper. Journal of Advanced Nursing-Institutional Subscription, 19(2), 328-335.

Barron, J. M. (2009). Masculinity ideology, gender role strain, and identity styles in male college students. University of South Dakota.

Beasley, C. (2008). Rethinking hegemonic masculinity in a globalizing world. Men and Masculinities, 11(1), 86-103.

Bewick, B. M., Mulhern, B., Barkham, M., Trusler, K., Hill, A. J., \& Stiles, W. B. (2008). Changes in undergraduate student alcohol consumption as they progress through university. BMC Public Health, 8(1), 163.

Beynon, J. (2001). Masculinities and culture. Buckingham, USA: Open University Press.

Bourke, B. (2014). Positionality: Reflecting on the research process. The qualitative report, 19(33), 1-9.

Brickell, C. (2005). Masculinities, performativity, and subversion: A sociological reappraisal. Men and Masculinities, 8(1), 24-43. 
Bridges, T. (2013). Issues of intimacy, masculinity and ethnography. In Men, masculinities and methodologies (pp. 53-63). London: Palgrave Macmillan.

Brownhill, S., Wilhelm, K., Barclay, L., \& Schmied, V. (2005). 'Big build': hidden depression in men. Australian and New Zealand journal of Psychiatry, 39(10), 921931.

Brush, S. G. (1978). Nettie M. Stevens and the discovery of sex determination by chromosomes. Isis, 69(2), 163-172.

Campbell, H. (2000). The glass phallus: Public masculinity and drinking in rural New Zealand. Rural Sociology, 65(4), 562-581.

Capdeville, V. (2012). Gender at stake: the role of eighteenth-century London clubs in shaping a new model of English masculinity. Culture, Society \& Masculinities, 4(1).

Carter, M. J., \& Fuller, C. (2015). Symbolic interactionism. Sociopediaisa, 1, 1-17.

Connell, R. W. (2005). Masculinities. Cambridge: Polity Press.

Connell, R. W. (1997). Men, masculinities and feminism. Social Alternatives, 16(3), 7.

Connell, R. W., \& Messerschmidt, J. W. (2005). Hegemonic masculinity: Rethinking the concept. Gender \& society, 19(6), 829-859.

Connell, R. W. (2000). The men and the boys. Australia: University of California Press.

Connell, R. W. (1985). Theorising gender. Sociology, 19(2), 260-272.

Constas, M. A. (1992). Qualitative analysis as a public event: The documentation of category development procedures. American Educational Research Journal, 29(2), 253-266.

Courtenay, W. H. (2000). Constructions of masculinity and their influence on men's wellbeing: a theory of gender and health. Social Science \& Medicine, 50(10), 13851401 .

Demetriou, D. Z. (2001). Connell's concept of hegemonic masculinity: A critique. Theory and society, 30(3), 337-361.

Dodge, R., Daly, A. P., Huyton, J., \& Sanders, L. D. (2012). The challenge of defining wellbeing. International Journal of Wellbeing, 2(3).

Donaldson, M. (1993). What is hegemonic masculinity? Theory and Society, 22(5), 643657.

Donovan, J. (2012). The way of men. Oregan, USA: Dissonant Hum.

Dynel, M. (2008). No aggression, only teasing: The pragmatics of teasing and banter. Lodz Papers in Pragmatics, 4(2), 241-261.

Easthope, A. (1992). What a man's gotta do: The masculine myth in popular culture. New York, USA: Routledge. 
Eckes, T., \& Trautner, H. M. (Eds.). (2012). The developmental social psychology of gender: An integrative framework. The developmental social psychology of gender (1 st ed., pp 3-33). New York, USA: Psychology Press.

Eisenberg, D., Downs, M. F., Golberstein, E., \& Zivin, K. (2009). Stigma and help seeking for mental health among college students. Medical Care Research and Review, 66(5), 522-541.

Englar-Carlson, M., \& Kiselica, M. S. (2013). Affirming the strengths in men: A positive masculinity approach to assisting male clients. Journal of Counseling \& Development, 91(4), 399-409.

Finzen, A. (2017). Stigma and stigmatization within and beyond psychiatry. In W. Gaebel, W. Rossler \& N. Sartorius (Eds.), The Stigma of Mental Illness-End of the Story? (pp. 29-42). Zurich, Switzerland: Springer International Publishing.

Frese, M. (2015). Cultural practices, norms, and values. Journal of Cross-Cultural Psychology, 46(10), 1327-1330.

Gavala, J. R., \& Flett, R. (2005). Influential factors moderating academic enjoyment/motivation and psychological well-being for Maori university students at Massey University. New Zealand Journal of Psychology, 34(1), 52-57.

Gee, S., \& Jackson, S. J. (2012). Leisure corporations, beer brand culture, and the crisis of masculinity: the Speight's 'Southern Man'advertising campaign. Leisure Studies, 31(1), 83-102.

Gergen, K. J. (2015). Culturally inclusive psychology from a constructionist standpoint. Journal for the Theory of Social Behaviour, 45(1), 95-107.

Giazitzoglu, A., \& Down, S. (2017). Performing entrepreneurial masculinity: An ethnographic account. International Small Business Journal, 35(1), 40-60.

Goffman, E. (1976). Gender display. In Gender advertisements (pp. 1-9). London: Palgrave.

Goffman, E. (1963). Stigma; notes on the management of spoiled identity. Englewood Cliffs, N.J.: Prentice-Hall

Goffman, E. (1959). The presentation of self in everyday life. New York, USA: Doubleday.

Goffman, E. (1967). Where the action is. In E. Goffman (Ed.), Interaction Ritual (pp. 149270). New York: Doubleday.

Gouveia, V. V., Vione, K. C., Milfont, T. L., \& Fischer, R. (2015). Patterns of value change during the life span: Some evidence from a functional approach to values. Personality and Social Psychology Bulletin, 41(9), 1276-1290.

Graeber, D. (2001). Toward an anthropological theory of value: The false coin of our own dreams. New York: Springer.

Guest, G., Bunce, A., \& Johnson, L. (2006). How many interviews are enough? An experiment with data saturation and variability. Field Methods, 18(1), 59-82. 
Hall, E. (2018, April 19). Men's Only Med Group Hopes to Combat Mental Health.

Retrieved from: https://www.critic.co.nz/news/article/7472/mens-only-med-grouphopes-to-combat-mental-health

Harris III, F. (2010). College men's meanings of masculinities and contextual influences: Toward a conceptual model. Journal of College Student Development, 51(3), 297318.

Harris III, F. (2008). Deconstructing masculinity: A qualitative study of college men's masculine conceptualizations and gender performance. NASPA Journal, 45(4), 453474.

Hearn, J. (2004). From hegemonic masculinity to the hegemony of men. Feminist Theory, 5(1), 49-72.

Hein, W., \& O'Donohoe, S. (2014). Practising gender: The role of banter in young men's improvisations of masculine consumer identities. Journal of Marketing Management, 30(13-14), 1293-1319.

Hirose, I., \& Olson, J. (2015). Introduction to Value Theory. In The Oxford Handbook of Value Theory (pp. 1-9). New York, USA: Oxford University Press

Hokowhitu, B. (2004). Tackling Maori masculinity: A colonial genealogy of savagery and sport. The Contemporary Pacific, 16(2), 259-284.

Hong, L. (2000). Toward a transformed approach to prevention: Breaking the link between masculinity and violence. Journal of American College Health, 48(6), 269-279.

Hopkins, J. \& Riley, J. (1998). Blokes and sheds. Auckland, New Zealand: Harper Collins.

Howson, R. (2006). Challenging hegemonic masculinity. New York, USA: Routledge.

Hunt, H., Morgan, N., \& Teddy, L. (2001). Barriers to and supports for success for Maori students in the Psychology Department at the University of Waikato. Hamilton, New Zealand: Department of Psychology, University of Waikato.

Irwin, M. (2011). Transitional experiences of first year young males at university. Ako Aotearoa. Retrieved from: https://ako.ac.nz/assets/Knowledge-centre/RHPF-c28Transitional-experiences-of-first-year-males-atuniversity/c9fc627cb7/RESEARCH-REPORT-Transitional-Experiences-of-FirstYear-Young-Males-at-University.pdf

Itulua-Abumere, F. (2013). Understanding men and masculinity in modern society. Open Journal of Social Science Research, 1(2), 42-45.

Jackson, C., \& Dempster, S. (2009). 'I sat back on my computer... with a bottle of whisky next to me': Constructing 'cool' masculinity through 'effortless' achievement in secondary and higher education. Journal of Gender Studies, 18(4), 341-356.

Jackson, C. (2010). 'I've been sort of laddish with them... one of the gang': teachers' perceptions of 'laddish' boys and how to deal with them. Gender and Education, 22(5), 505-519. 
Johnston, L., Huggard, P. K., \& Goodyear-Smith, F. A. (2008). Men's health and the health of the nation. New Zealand Medical Journal, 121(1287), 69-76.

Joseph, J., \& Falcous, M. (2017). Negotiating the 'Kiwi Bloke': accessing mosaic masculinities through Afro-Brazilian sport in New Zealand/Aotearoa. Qualitative Research in Sport, Exercise and Health, 11(2), 1-15.

Kennedy, J. C. (2007). Leadership and culture in New Zealand. Culture and Leadership Across the World: The GLOBE Book of In-Depth Studies of 25 Societies, 397-432.

Keyes, C. L. (Ed.). (2012). Mental well-being: International contributions to the study of positive mental health. Springer Science \& Business Media.

Kightley, O. (2018, August 26). Oscar Kightley: New Zealand, we have a problem. Retrieved from https://www.stuff.co.nz/national/health/106567003/oscar-kightleynew-zealand-we-have-a-problem

King, M. (Ed.). (1988). One of the Boys? Changing Views of Masculinity in New Zealand. Auckland: Heinemann.

King, M. (2003). Penguin History of New Zealand. UK: Penguin.

Kirkland, A. (2014). What is wellness now? Journal of Health Politics, Policy and Law, 39(5), 957-970.

Kirkpatrick, M. (2003). The nature and nurture of gender. Psychoanalytic Inquiry, 23(4), 558-571.

Kroeber, A. L., \& Kluckhohn, C. (1952). Culture: A critical review of concepts and definitions. Papers of the Peabody Museum of Archaeology \& Ethnology, Harvard University, 47(1)

La Placa, V., McNaught, A., \& Knight, A. (2013). Discourse on wellbeing in research and practice. International Journal of Wellbeing, 3(1), 116-125.

Law, R. (1997). Masculinity, place, and beer advertising in New Zealand: The Southern Man campaign. New Zealand Geographer, 53(2), 22-28.

Lipson, S. K., \& Eisenberg, D. (2018). Mental health and academic attitudes and expectations in university populations: results from the healthy minds study. Journal of Mental Health, 27(3), 205-213.

Lomas, T., Cartwright, T., Edginton, T., \& Ridge, D. (2016). New Ways of Being a Man: "Positive" Hegemonic Masculinity in Meditation-based Communities of Practice. Men and Masculinities, 19(3), 289-310.

Lomas, T. (2013). Critical positive masculinity. Masculinities \& Social Change, 2(2), 167193.

Longhurst, B., Smith, G., Bagnall, G., Crawford, G., Ogborn, M., Baldwin, E., \& McCracken, S. (2017). Introducing Cultural Studies. London, UK: Routledge.

Long, J. (2018, August 22). Uni students demand better mental health support from government. Retrieved from: 
https://www.stuff.co.nz/national/health/106489293/uni-students-demand-bettermental-health-support-from-government

Manwell, L. A., Barbic, S. P., Roberts, K., Durisko, Z., Lee, C., Ware, E., \& McKenzie, K. (2015). What is mental health? Evidence towards a new definition from a mixed methods multidisciplinary international survey. BMJ open, 5(6), e007079.

Martin, D. D., \& Wilson, J. L. (2005). Role theory. Encyclopaedia of Social Theory, 651655.

Marvelly, L. (2019, September 21). Lizzie Marvelly: Support the All Blacks and your 'team' at home as well. Retrieved from: https://www.nzherald.co.nz/nz/news/article.cfm?c_id=1\&objectid=12269012

Masika, R., \& Jones, J. (2016). Building student belonging and engagement: Insights into higher education students' experiences of participating and learning together. Teaching in Higher Education, 21(2), 138-150.

McGuigan, J. (Ed.). (1997). Cultural methodologies. London, UK: Sage publications.

McKenzie, S. K., Collings, S., Jenkin, G., \& River, J. (2018). Masculinity, Social Connectedness, and Mental Health: Men's Diverse Patterns of Practice. American Journal of Men's Health, 12(5), 1247-1261.

McKenzie, S. (2017). Understanding Men's Mental Health: Gender Relations and Mental Well-Being (Thesis, Doctor of Philosophy). University of Otago. New Zealand. Retrieved from http://hdl.handle.net/10523/7572

McPhee, E. (2019). Uni women outnumber men 60:40. Retrieved from:

https://www.odt.co.nz/news/dunedin/campus/university-of-otago/uni-women-outnumbermen-6040

Ministry of Justice. (2018). Provisional Suicide Figures- August 2018. Retrieved March 05, 2019, from https://coronialservices.justice.govt.nz/suicide/annual-suicide-statisticssince-2011/

Morgan, G. (2006). Images of Organization (Updated Edition). California, USA: Sage Publications.

Murphy, J. G., McDevitt-Murphy, M. E., \& Barnett, N. P. (2005). Drink and be merry? Gender, life satisfaction, and alcohol consumption among college students. Psychology of Addictive Behaviors, 19(2), 184.

Nelson, C., Treichler, P. A., \& Grossberg, L. (1992). Cultural studies: An introduction. Cultural studies, 1(5), 1-19.

New Zealand Union of Students Associations. (2018). Kei Te Pai? Report on Student Mental Health in Aotearoa. Retrieved from: https://gallery.mailchimp.com/b109fde7924adea2d9afaa28d/files/3d3cdb2b-c0ef4191-847e-3f32b0bf21eb/Kei_Te_Pai_Report_on_Student_Mental_Health.pdf

Oliffe, J. L., \& Phillips, M. J. (2008). Men, depression and masculinities: A review and recommendations. Journal of Men's Health, 5(3), 194-202. 
Oransky, M., \& Marecek, J. (2009). "I'm not going to be a girl": masculinity and emotions in boys' friendships and peer groups. Journal of Adolescent Research, 24(2), 218241.

Penwarden, S. (2010). Re-thinking the Kiwi bloke: Young men's stories of resistance to drinking alcohol as a way of performing masculinity. Explorations: An E-Journal of Narrative Practice, 1, 43-56.

Pickering, M. (Ed.). (2008). Research methods for cultural studies. Edinburgh, UK: Edinburgh University Press.

Pitman, A., Krysinska, K., Osborn, D., \& King, M. (2012). Suicide in young men. The Lancet, 379(9834), 2383-2392.

Pleck, J. H. (2018). The theory of male sex-role identity: Its rise and fall, 1936 to the present. In H. Brod (Eds.), The Making of Masculinities (Routledge Revivals) (pp. 21-38). London, UK: Routledge.

Pollack, William S. (1999). Real Boys: Rescuing Our Sons from the Myths of Boyhood. New York, USA: Henry Holt \& Company

Robertson, S. (2007). Understanding men and health: Masculinities, identity and wellbeing. Berkshire, UK: Open University Press.

Sardar, Z. (2004). Introducing cultural studies: A graphic guide. Cambridge, UK: Icon Books.

Sayette, M. A., Creswell, K. G., Dimoff, J. D., Fairbairn, C. E., Cohn, J. F., Heckman, B. W. \& Moreland, R. L. (2012). Alcohol and group formation: A multimodal investigation of the effects of alcohol on emotion and social bonding. Psychological science, 23(8), 869-878.

Sayette, M. A. (2017). The effects of alcohol on emotion in social drinkers. Behaviour Research and Therapy, 88, 76-89.

Schein, E. H. (2010). Organizational culture and leadership (Fourth Edition). San Fransisco, USA: Jossey-Bass.

Schwalbe, M., \& Wolkomir, M. (2001). The masculine self as problem and resource in interview studies of men. Men and Masculinities, 4(1), 90-103.

Schwartz, S. H. (2012). An Overview of the Schwartz Theory of Basic Values. Online Readings in Psychology and Culture, 2(1), 3-20.

Seidler, Z. E., Rice, S. M., River, J., Oliffe, J. L., \& Dhillon, H. M. (2018). Men's mental health services: The case for a masculinities model. The Journal of Men's Studies, 26(1), 92-104.

Seligman, M. E., \& Csikszentmihalyi, M. (2014). Positive psychology: An introduction. In M. Csikszentmihalyi, Flow and the foundations of positive psychology (pp. 279298). California, USA: Springer, Dordrecht. 
Shpancer, N. (2011, August 4). Male and Female: The Overlapping Curves. Retrieved from: https://www.psychologytoday.com/us/blog/insight-therapy/201108/male-andfemale-the-overlapping-curves

Sim, J., \& Thomson, R. (2000, August 8). Hegemonic Masculinity in New Zealand: The Reconstruction of the 'Kiwi Bloke'. Retrieved from: https://www.researchgate.net/publication/280553864_Hegemonic_masculinity_in_ New_Zealand_The_reconstruction_of_the_kiwi_bloke

Skerrett, A. (2019, June 16). Men's Health Week: 'Everyone's got a story to tell'-All Black's mental health crusade. Retrieved from: https://www.newshub.co.nz/home/rural/2019/06/men-s-health-week-everyone-sgot-a-story-to-tell-all-black-s-mental-health-crusade.html

Slade, M. (2010). Mental illness and well-being: the central importance of positive psychology and recovery approaches. BMC health services research, 10(1), 26.

Smiler, A. P. (2004). Thirty years after the discovery of gender: Psychological concepts and measures of masculinity. Sex Roles, 50(1-2), 15-26.

Smith, G. (1990). Will the real Mr. New Zealand please stand up? Australia: Penguin Books.

Spates, J. L. (1983). The sociology of values. Annual Review of Sociology, 9(1), 27-49.

Spector-Mersel, G. (2006). Never-aging stories: Western hegemonic masculinity scripts. Journal of Gender Studies, 15(1), 67-82.

Stern, C. (2016). Undoing insularity: A small study of gender sociology's big problem. Econ Journal Watch, 13(3), 452-466.

Storrie, K., Ahern, K., \& Tuckett, A. (2010). A systematic review: Students with mental health problems - a growing problem. International Journal of Nursing Practice, 16(1), 1-6.

Tagg, B. (2008). Imagine, a Man Playing Netball! Masculinities and Sport in New Zealand. International Review for the Sociology of Sport, 43(4), 409-430.

Tanner, J. L., \& Arnett, J. J. (2011). Presenting "emerging adulthood": What makes it developmentally distinctive (pp. 13-30). In J.J. Arnett, M. Kloep, L.B. Hendry, J. Tanner, Debating Emerging Adulthood: Stage or Process. New York, USA: Oxford University Press.

Tata, J. R. (2005). One hundred years of hormones: A new name sparked multidisciplinary research in endocrinology, which shed light on chemical communication in multicellular organisms. EMBO Reports, 6(6), 490-496.

Taylor, S. J., Bogdan, R., \& DeVault, M. (2015). Introduction to qualitative research methods: A guidebook and resource. USA: John Wiley \& Sons.

Tertiary student leaders call for action after research shows many struggle with mental health. (2018, July 11). NZ Herald. Retrieved from: https://www.nzherald.co.nz/nz/news/article.cfm?c_id=1\&objectid=12086535 
Thomas, L. (2012). Building student engagement and belonging in Higher Education at a time of change. UK: Paul Hamlyn Foundation.

Tov, W., \& Diener, E. (2009). Culture and subjective well-being. In E. Diener, E.M. Suh, Culture and well-being (pp. 9-41). London, UK: The MIT Press.

Udry, J. R. (1994). The nature of gender. Demography, 31(4), 561-573.

Wassersug, R. J., \& Hamilton, L. D. (2018). Masculinity in Milliseconds: An Evolutionary \& Neurophysiological Perspective on Expressions of Masculinity. Adaptive Human Behavior and Physiology, 4(2), 152-170.

Way, N. (2011). Deep secrets. London, UK: Harvard University Press.

Wenner, L. A., \& Jackson, S.J. (2009). Sport, beer, and gender: Promotional culture and contemporary social life. New York, USA: Peter Lang.

West, C., \& Zimmerman, D. H. (1987). Doing gender. Gender \& Society, 1(2), 125-151.

Westerhof, G. J., \& Keyes, C. L. (2010). Mental illness and mental health: The two continua model across the lifespan. Journal of adult development, 17(2), 110-119.

Wharton, A. S. (2009). The sociology of gender: An introduction to theory and research. Oxford, UK: John Wiley \& Sons.

Willott, S., \& Lyons, A. C. (2012). Consuming male identities: Masculinities, gender relations and alcohol consumption in Aotearoa New Zealand. Journal of Community \& Applied Social Psychology, 22(4), 330-345.

Woosler, M. (2018). Fighting America's Loneliness Problem. Retrieved from: https://capitalresearch.org/article/fighting-americas-loneliness-problem/ 


\title{
Appendix A
}

\author{
Interview Guide \\ Introductory questions \\ 1. What are you studying? \\ 2. How long have you been studying it? \\ 3. Have you lived in New Zealand all your life? If not, how long have you been here?
}

\section{Masculinity}

4. How would you describe an "average kiwi man"?

5. What are some of the things that this man does?

6. What do you think people value in this kind of man?

7. Do you think that there are expectations that men conform to these practices and values?

Mental Wellbeing

8. What do you think "mental wellbeing" is?

9. Do you think any of the values and practices we discussed are good for mental wellbeing? If yes, in what way?

10. Do you think any of the values and practices we discussed are bad for mental wellbeing? If yes, in what way?

11. Do you think being a student has an effect on mental wellbeing?

12. Do you think being both a student and a guy in New Zealand effects mental wellbeing? 


\section{Appendix B}

\section{Participants Needed \\ Each participant will go in a draw to win $\$ 50$ New World Voucher}

Exploring views on masculinity and mental wellbeing: The case of male students in higher education New Zealand

I am a Master's student conducting research on views on masculinity in New Zealand and how it shapes perceptions of mental wellbeing. I am looking for participants who are willing to be interviewed on this topic. Interviews will take approximately one hour, and you will be able to withdraw from the interview at any point. There will be a series of open-ended questions that will invite you to share your views on various points surrounding the above topic.

To participate, you must: identify as male between the ages of $18-25$ and be enrolled in either an undergraduate or postgraduate program at a tertiary institution in New Zealand.

This research, supervised by Dr Sally Riad, has been approved by the Victoria University Human Ethics Committee.

I will be conducting interviews for this study until May $1^{\text {st }} 2019$.

Please contact me via the email provided below for further information or to express your interest in participation.

Thank You!

\begin{tabular}{|c|c|c|c|c|c|c|c|c|c|}
\hline 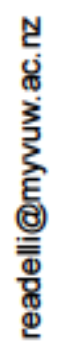 & 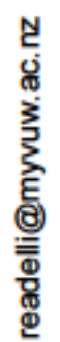 & 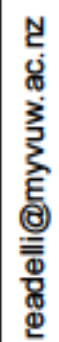 & 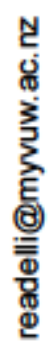 & 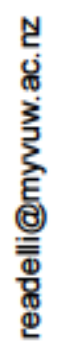 & 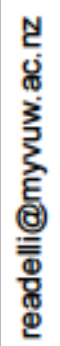 & 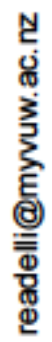 & 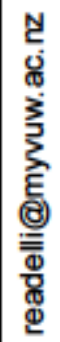 & 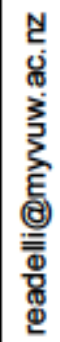 & 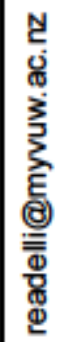 \\
\hline
\end{tabular}




\section{Appendix C}

SCHOOL OF MANAGEMENT

TE KURA TLAKL, WHAKAWHITI KÖRESO

LEVEl 10, RUTHERFORD HOUSE, PIPITEA CAMPUS, 23 LAMBTON QUAY, WELUNGTON

PO Box 600, Wellington 6140, New Zealand

\section{Participant Information Sheet}

Research Project Title: Exploring views on masculinity and mental wellbeing: The case of male students in higher education in New Zealand.

Thank you for considering to participate in this study,

This study explores views on masculinity in New Zealand and how it shapes perceptions of mental wellbeing. Victoria University requires, and has granted, approval from the schools Human Ethics Committee. The application number is 0000026941

I am inviting male students between the ages of 18-25 who are currently enrolled at a tertiary institution in New Zealand to participate in this research. As a participant you will be invited to address questions on the above topic in an interview that will take approximately one hour. Permission will be asked to audio record the interview, and a summary of the interview will be sent to participants upon request. If at any point you should wish to stop the interview you may do so.

You will not be identified personally in any written report produced from this research, including possible publication in academic conferences and journals. All material collected will be kept confidential and will be viewed only by myself and my supervisor Dr Sally Riad. The thesis will be submitted for marking to the School of Management, and subsequently deposited in the University Library. Should any participant wish to withdraw from the project, they may do so two weeks from the completion of their interview. Their data collected up to that point will be destroyed. All data collected from participants will be destroyed within two years after the completion of the project.

If you have any questions or would like to receive further information about the project after this interview, please contact me through the email address provided below.

Researcher: Ellis Read

Master of Commerce

Victoria university of Wellington

readelli@myvuw.ac.nz

Supervisor: Dr Sally Riad

Senior lecturer School of Management

Victoria University of Wellington

Sally.Riad@vuw.ac.nz

044635079 


\section{Appendix D}

SCHOOL OF MANAGEMENT

TE KURA TIAKL, WHAKAWHITI KÖFERO

LEVE. 10, RUTHERFORD HOUSE, PIPITEA CAMPUS, 23 LANBTON QUAY, WELUNGTON

PO Box 600, Wellington 6140, New Zealand

\section{Participant Consent Form}

Research Project Title: Exploring views on masculinity and mental wellbeing: The case of male students in higher education New Zealand

Researcher: Ellis Read, School of Management, Victoria University of Wellington

I have been given and have understood an explanation of this research project. I have had an opportunity to ask questions and have them answered to my satisfaction.

I understand that I may withdraw myself (or any information I have provided) from this project, without having to give reasons, by e-mailing readelli@myvuw.ac.nz by the $30^{\text {th }}$ March 2019.

I understand that any information I provide will be kept confidential to the researcher and their supervisor, the published results will not use my name, and appropriate efforts will be made to limit information that could pertain to my identity.

I understand that the data I provide will not be used for any other purpose beyond academic research and possible publication or released to anyone other than the researcher and supervisor.

I understand that, if this interview is audio recorded, the recording and transcripts of the interviews will be erased within two years after the conclusion of the project. Furthermore, I will also have an opportunity to view a summary of the interview.

Please indicate (by ticking the boxes below) which of the following apply:

I would like to receive a summary of the results of this research when it is completed.

I agree to this interview being audio recorded.

I agree to being interviewed without audio recording

Signed:

Name of participant:

Date:

Email (if summary is requested): 